

\title{
Tous aux abris! Les occupations du Paléolithique final et du Mésolithique dans les cavités naturelles du Massif armoricain
}

Grégor Marchand, Nicolas Naudinot

\section{- To cite this version:}

Grégor Marchand, Nicolas Naudinot. Tous aux abris! Les occupations du Paléolithique final et du Mésolithique dans les cavités naturelles du Massif armoricain. Bulletin de la Société préhistorique française, 2015, 112 (3), pp.517-542. hal-02438613

\section{HAL Id: hal-02438613 https://hal.science/hal-02438613}

Submitted on 14 Jan 2020

HAL is a multi-disciplinary open access archive for the deposit and dissemination of scientific research documents, whether they are published or not. The documents may come from teaching and research institutions in France or abroad, or from public or private research centers.
L'archive ouverte pluridisciplinaire HAL, est destinée au dépôt et à la diffusion de documents scientifiques de niveau recherche, publiés ou non, émanant des établissements d'enseignement et de recherche français ou étrangers, des laboratoires publics ou privés. 


\title{
Tous aux abris!
}

\section{Les occupations du Paléolithique final et du Mésolithique dans les cavités naturelles du Massif armoricain}

\author{
Grégor MARCHAND et Nicolas NAUDiNOT
}

Résumé : Si elles sont communes et même emblématiques dans le domaine de la Préhistoire, les fouilles dans les grottes ou abris-sous-roche sont très rares sur le Massif armoricain pour le Paléolithique final et le Mésolithique. Les travaux de terrain menés depuis 2007 dans certaines cavités du Finistère (Pont-Glas à Plounéour-Ménez, Kerbizien à Huelgoat et le Rocher de l'Impératrice à Plougastel-Daoulas) offrent un nouvel éclairage sur les manières d'habiter des espaces restreints. Une nouvelle étude est également proposée du mobilier de la seule grotte continentale de Bretagne, Roc'h Toul à Guiclan (Finistère), fouillée de manière pour le moins expéditive en 1868. Une première typologie de ces cavités et de leur remplissage pu être ensuite établie pour certains substrats géologiques seulement (granites et quartzites). À l'exception notable de la grotte marine effondrée de Ménez Drégan à Plouhinec (Finistère) occupée au Paléolithique ancien, la modicité des remplissages sédimentaires des cavernes armoricaines ne donne pas prise à des modélisations chronologiques de grande ampleur. L'intérêt qu'on leur porte est davantage dans la complémentarité fonctionnelle que leurs contenus archéologiques nous offrent en regard des sites de plein air. Dans le mobilier lithique, les phases d'usage et de réfection de l'outillage sont clairement surreprésentées, au détriment des phases de production. Cela plaide pour des usages logistiques de courte durée, dans des systèmes de mobilité différents, plus amples au Paléolithique final qu'au Mésolithique. Malgré leurs limites que cet article s'emploie à bien définir, ces premières fouilles ont bel et bien livré des signatures fonctionnelles très originales dans l'outillage ou dans les manifestations symboliques, qui engagent à réfléchir au rôle de ces petites cavernes dans les réseaux d'habitats du Paléolithique final et du Mésolithique.

Mots-clés : abris-sous-roche, Azilien, Bretagne, Mésolithique, mobilité, Paléolithique final, paléo-économie.

\begin{abstract}
While common and even iconic in the field of Prehistory, excavations in caves or rock-shelters are very rare in the Armorican Massif for the final Palaeolithic and Mesolithic. Why are these sites largely ignored by research in this part of France, when they form the basis of archaeological work in other parts of Europe? Are rock-shelters missing in Brittany? Of course not... Rock-shelters are actually numerous in Brittany. These sites differ however from the large caves of southern France. They have been neglected because of their small size and the shallow depth of their sedimentary deposits, and also because they do not correspond to the romantic idea people have of caves and rockshelters... This particular situation is essentially due to the geological context of Brittany, where the crystalline massif contains no limestone. Rock shelters are thus found in various specific contexts: naturally supported granite boulders, the foot of sandstone or schist cliffs, or marine erosion faults in granite, sandstone or schist contexts.

A new collective research programme, focused on the Late glacial and the Mesolithic, is now aiming to integrate these little known sites with a view to gaining a better understanding of the development the prehistoric palaeo-economic system. While much information has been obtained about Late glacial and Mesolithic societies in this part of Europe over the last twenty years, it essentially comes from large open-air sites that do not reflect the broad spectrum of site types making up the prehistoric palaeo-economic system. Whatever the dominant mobility system of a hunter-gatherer group, small sites are numerous and are essential to understanding the complexity of the system. These small sites are generally invisible in survey or are not examined by preventive archaeology. Investigating rock-shelters appears to be an excellent and easy way to study small sites since they have very small surface areas and are particularly easy to spot in the landscape. Since the socio-economic systems vary throughout Prehistory, our diachronic approach, which considers different environments and climates, enables us to study the development of cultural systems through time and examine how rock-shelter use changed over this long period. Although this research programme has just started, fieldwork has already been conducted in Finistère by the two authors of this paper (Pont-Glas in Plounéour-Menez, Kerbizien Huelgoat and the Rocher de l'Impératrice in Plougastel-Daoulas).

These sites offer a new insight into ways of living in small spaces. The first site is a fallen block shelter, very common in the granitic massif of Brittany. The site was first excavated in 2007 and 2008. Two main Mesolithic occupations have been identified, as well as a later occupation dated to the Iron Age (around 300 BC). The spatial analysis of the site, together with the study of the lithic assemblage, suggest that Pont-Glas is a logistic site where hunters or warriors
\end{abstract}


sheltered over a short period. Kerbizien is another rock-shelter in a granitic context. The excavation carried out in July 2011 identified Tardiglacial (Azilian) levels at the back of the cave in a two metres wide band along the wall. The Early Azilian lithic industry is made up of curved back points, very utilized large blades and burin spalls. Its functional signature is original and seems quite compatible with the hypothesis of a logistic station (i.e. hunting camp) for very mobile human groups. Their knowledge of the local geological resources is a significant aspect to be kept in mind, showing that hunts were regularly made in this area.

Excavations at the Rocher de l'Impératrice are still in progress. This rock-shelter, at the foot of a high sandstone cliff overlooking the Brest roadstead, was mainly occupied during the Early Azilian. The evidence suggests this site was used by small groups of people for short periods. Activities seem to have focused essentially on game acquisition and processing. Yet contrary to Pont-Glas, there is some evidence for other activities in addition to hunting. The discovery of several dozen engraved (and sometimes coloured) schist tablets seems to point in this direction, indicating manufacture or/and use of these 'symbolic' elements during the occupation of the shelter by the Early Azilians. Despite the limitations of their archaeological context, these initial excavations produced original functional signatures in tool-kits and symbolic activities, highlighting the role of small caves in settlement networks during the final Palaeolithic and the Mesolithic. These results contribute to a first attempt at modelling the use of rock-shelters in Brittany and the evolution of paleo-economic systems between the Late Glacial and the Late Mesolithic.

Keywords: rock-shelter, Azilian, Brittany, Mesolithic, mobility, Late Palaeolithic, palaeo-economy.

Au lieu des rêves évasifs, toujours infortunés, qu'on désigne comme des châteaux en Espagne, la grotte est un rêve concentré.

Gaston Bachelard

La Terre et les rêveries du repos, 1948, p. 214

\section{L'IMMARCESCIBLE ATTRAIT DES CAVERNES}

L' exploration d'occupations humaines en abris-sousroche semble si banale pour l'archéologie préhistorique qu'il peut sembler étonnant d'y consacrer quelques pages de synthèse en ce début de troisième millénaire et encore plus d'esquisser un programme d'étude spécifique pour les années à venir. Ce n'est pourtant pas une voie de recherche commune sur le Massif armoricain pour la Préhistoire récente, à cause des apports scientifiques jugés somme toute modestes de ces pièges sédimentaires en regard des habitats de plein air. Les prospections d'envergure menées sur les sites mésolithiques en Bretagne et en Pays de la Loire à partir des années 1970 se sont en effet soldées par des bases de données garnies de centaines de sites ou d'indices de sites (Collectif, 1984; Gouraud, 1995; Gouletquer et al., 1996; Marchand, 2009). Les pièces lithiques récoltées y atteignent parfois plusieurs centaines de milliers d'éléments. Au contraire du Bassin parisien ou de l'Est de la France, ces sites de plein air ont été assez timidement abordés par l'archéologie préventive (Tinévez et al., 1987; Blanchet et al., 2002; Nicolas et al., 2012). Plusieurs sondages programmés ont aidé à en comprendre les limites sédimentaires, soulignant notamment la difficulté à y trouver des césures spatiales pertinentes ou des stratigraphies (Marchand, 2005). Pour le Tardiglaciaire, c'est à l'inverse une fouille préventive menée sur une grande superficie aux Chaloignes, Mozésur-Louet, Maine-et-Loire, qui a donné une impulsion décisive aux recherches (Marchand et al., 2009). Elles aussi sont restées concentrées sur les habitats de plein air, les plus nombreux et les plus aisés à identifier (Marchand et al., 2004). Pour l'Azilien, on dispose désormais dans la documentation de petits locus au sein d'un même habitat, au côté d'une soixantaine de sites identifiés par ramassage de surface. Leurs limites spatiales sont évidemment fort limitées et mal estimées; leur chronologie est encore plus évanescente lorsqu'il s'agit d'aller au-delà du constat des présences-absences. À ces sites s'ajoutent une dizaine d'habitats post-aziliens, qu'il est possible de placer entre la seconde moitié du Dryas récent et le début du Préboréal (Naudinot, 2010 et 2013), dont le grand gisement de plein air de la Fosse à Villiers-Charlemagne en Mayenne récemment fouillé (Naudinot et Jacquier, 2009, 2013a et 2014; Jacquier et Naudinot, 2015).

L'élaboration de modèles paléo-économiques a suivi logiquement cette phase d'inventaire et de caractérisation technotypologique des industries lithiques, tant pour le Paléolithique final (Naudinot, 2010 et 2013a; Naudinot et Jacquier, 2014; Jacquier et Naudinot, 2015) que pour le Mésolithique (Marchand, 2005, 2012 et 2014; Yven, 2003a et b). Pour ces deux périodes, ils ont été réalisés à partir des données sur les territoires d'acquisition, les structures d'habitat ou encore la composition fonctionnelle de l'outillage; ce n'est que pour le Mésolithique que nous bénéficions des nombreux restes organiques issus des amas coquilliers du littoral atlantique, qui permettent d'approcher les rythmes de la mobilité ou la nature des proies capturées. Mais ces études achoppent sur l'importance quantitative des corpus, fruit à la fois du statut fonctionnel de ces habitats pérennes et des multiples réoccupations souvent sans césures sédimentaires. Les multiples apports d'outils au cours du temps ne peuvent être discriminés, non plus que l'extension de chaque campement; comment alors traiter de leurs complémentarités au sein des réseaux économiques et des territoires? La recherche de petites stations est devenue un axe crucial des travaux de terrain (Marchand, 2007; Marchand et Le Goffic, 2009). L'archéologie préventive ne 
parvenant pas dans ces régions à isoler de petites occupations en fond de vallée, nous avons logiquement tourné nos regards vers des contextes restreints par leur nature même, les abris-sous-roche.

Les domaines géologiques plutoniques ou métamorphiques du Massif armoricain génèrent essentiellement des cavités de modeste superficie, avec des remplissages plus ou moins importants suivant la nature de l'encaissant et l'orientation de la cavité. Ils n'ont cependant jamais livré de séquences stratigraphiques très développées pour la Préhistoire récente, au contraire du Paléolithique ancien et moyen qui est documenté par quelques abrissous-roche, des pieds de rochers ou des couloirs d'érosion marine (Monnier, 1980), sans que cela représente toutefois un potentiel équivalent à celui de certains bassins sédimentaires. C'est d'ailleurs cette discrétion qui a assuré la préservation du contenu des cavernes armoricaines. Les fouilles récentes menées dans le Finistère sur les sites de Pont-Glas (Plounéour-Ménez), Kerbizien (Huelgoat) et le Rocher de l'Impératrice (Plougastel-Daoulas), de même que des prospections encore aléatoires dans la région Bretagne, nous permettent aujourd'hui de dresser un bilan de ce potentiel en cavités, en particulier dans les formations de granite ou de grès-quartzites (fig. 1). En effet les karsts sont exceptionnels dans le nord-ouest de la France et ils sont creusés aux dépens de calcaires d'âge primaire dans des enclaves, ainsi à l'est dans la vallée de l'Erve (Mayenne) et du Layon (Maine-et-Loire), ou plus à l'ouest autour de Plougastel-Daoulas (Finistère); ils ne seront pas abordés directement dans cet article.

Malgré leurs limites que nous nous employons à bien définir, ces premières fouilles ont bel et bien livré des signatures fonctionnelles très originales dans l'outillage ou dans les manifestations symboliques, qui engagent à réfléchir au rôle de ces petites cavernes dans les réseaux d'habitats du Paléolithique final et du Mésolithique.

\section{UNE COURTE HISTOIRE DES RECHERCHES SUR LES CAVERNES ARMORICAINES}

$E^{n}$ n dépit d'un potentiel tout relatif en cavités profondes, la recherche sur les habitats du Paléolithique commença en Bretagne par une fouille en grotte! Ici comme dans le reste du monde, il semble bien que le préhistorien soit prisonnier de son rêve de la grotte originelle ou du mythe de la caverne... C'est donc le 4 juin 1868 que le Dr M. Le Hir entama ses recherches dans la caverne de Roc'h Toul à Guiclan (Finistère). Cette première fouille, bien qu'expéditive, avait alors permis de découvrir « les premières traces de ces hommes primitifs sur notre sol » (Le Hir, 1874, p. 90) sous la forme d'une industrie lithique aujourd'hui rattachée essentiellement à la phase ancienne de l'Azilien (Marchand et al., 2004; Naudinot, 2010). Les recherches sur les cavités du Massif armoricain s'éteignirent juste après la vidange de cette grotte et avec elles, les travaux sur le Paléolithique, mal- gré quelques rares découvertes fortuites attribuables au Paléolithique comme le gisement de plein air moustérien du Mont-Dol (Sirodot, 1873, cité par Monnier, 1980). En 1915, une « fouille » à l'aide de « leviers de pneu » a été réalisée dans un abri-sous-roche en chaos granitique à Locunolé (Finistère), dans les impressionnantes gorges des Roches du Diable que franchit la rivière Ellé. L'enthousiaste archéologue décrivit de manière sommaire dans les pages du Bulletin de la Société préhistorique française les silex et les pierres de foyer qui gisaient sous $35 \mathrm{~cm}$ de sédiment, avec une attribution au Néolithique (Boismoreau, 1915). L'apport scientifique réel de cette opération se résume désormais à un point de localisation sur une carte, ce qui illustre assez les ravages irrémédiables occasionnés aux fragiles remplissages de nombreuses cavités. Et encore, celle-ci a-t-elle été signalée !

Il faudra ensuite attendre les années 1950 et le développement de l'archéologie moderne dans l'Ouest de la France pour que de nouvelles cavités paléolithiques soient explorées. Ce fut d'abord le cas dans le sud-est du Massif armoricain sur le site de Roc-en-Pail, Chalonnessur-Loire, Maine-et-Loire. Prospecté par M. Gruet à partir de 1935 et fouillé en 1950, ce gisement présente une importante stratigraphie comprenant différents technocomplexes moustériens, avec au sommet des niveaux résiduels du Paléolithique supérieur ancien (Visset, 1979; Gruet, 1984). La nature de l'abri-sous-roche est aujourd'hui difficile à comprendre du fait de travaux d'extraction de chaux dans le secteur; son existence est d'ailleurs uniquement renseignée par la tradition orale et par quelques textes anciens.

Ce sont les falaises marines et dans une moindre mesure celles des estuaires qui ont livré les principales cavités paléolithiques explorées après la seconde guerre mondiale. Ainsi le long de la Rance - fleuve bretillien P.-R. Giot découvrit et fouilla l'abri-sous-roche moustérien de Grainfollet à Saint-Suliac (Giot et Bordes, 1955). $\mathrm{Au}$ pied de cette paroi de schiste briovérien relativement peu inclinée, la zone couverte n'était profonde que d'environ six mètres et le double surplomb était très élevé. À partir des années 1970, de nombreux autres gisements paléolithiques furent découverts en Bretagne (Monnier, 1980). Malgré cette dynamique de recherche, les sites en abris ou en grottes restaient rares. La plupart des gisements découverts durant ces années sont en effet localisés sur des plages anciennes ou dans des falaises marines. L'abrisous-roche moustérien de Goaréva (île de Bréhat, Côtesd'Armor) est considéré comme le plus emblématique pour le domaine côtier en Bretagne. Il a été découvert par P.-R. Giot en 1973 puis fouillé par J.-L. Monnier (Giot et Monnier, 1975; Monnier, 1988). Sur le littoral du Sud de la Bretagne, Saint-Colomban, Carnac, Morbihan (Monnier et Le Cloirec, 1985) et Menez Drégan, Plouhinec, Finistère (Hallegouët et al., 1992; Laforge et Monnier, 2011) sont des couloirs d'érosion marine, des failles qui se terminent par des abris ou de petites grottes occupées au Paléolithique ancien, comme l'ont montré les fouilles de J.-L. Monnier et son équipe (Monnier, 1988 et 2006). En Basse-Normandie, c'est l'abri-sous-roche du Rozel, dans 


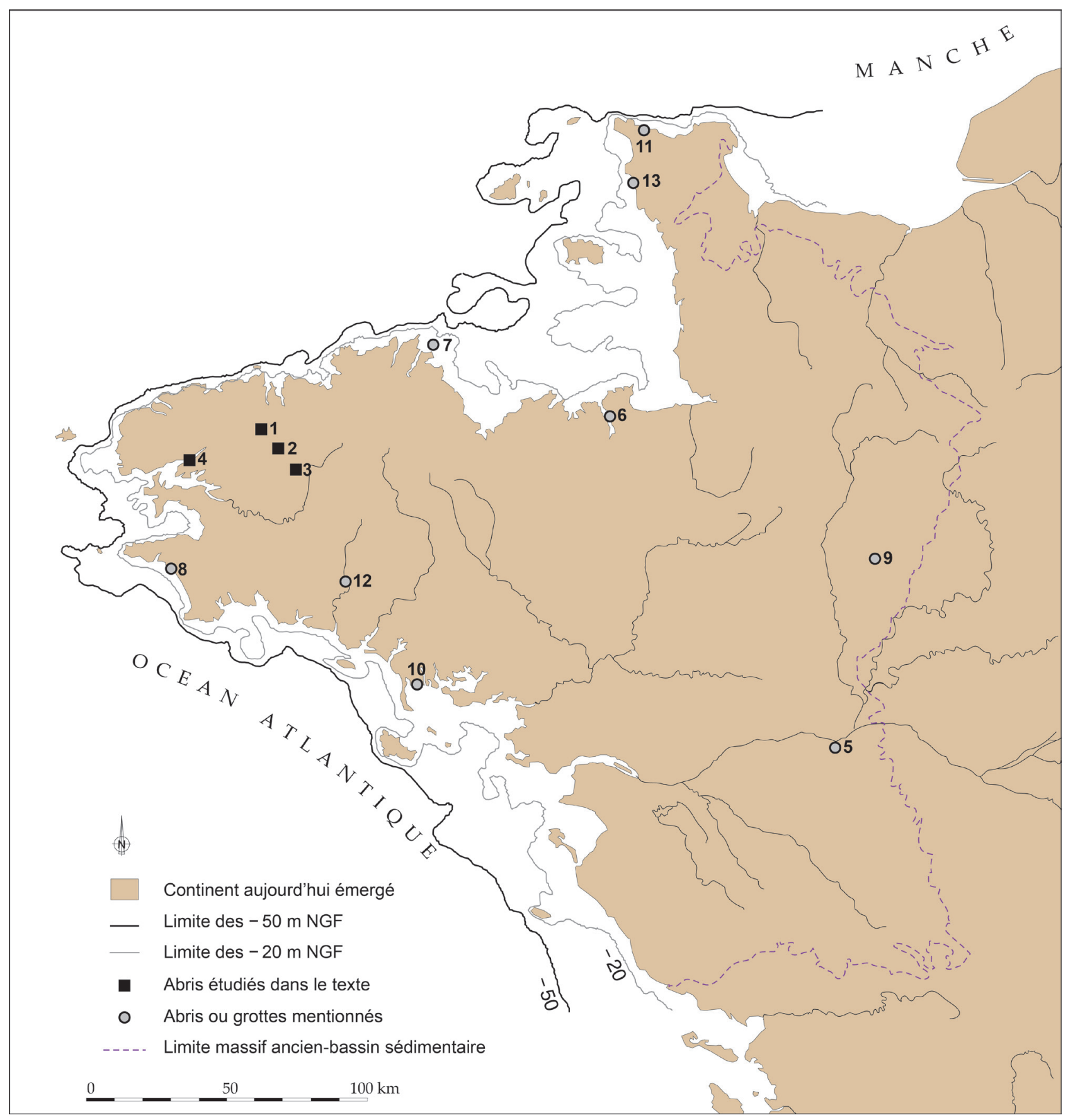

Fig. 1 - Carte des sites mentionnés dans le texte (points gris), avec indication des quatre abris du Paléolithique final et du Mésolithique objets de cet article (carrés noir). La courbe bathymétrique des $-50 \mathrm{~m}$ évoque le rivage à la fin du Tardiglaciaire, celle des $-20 \mathrm{~m}$ le rivage vers le VIII ${ }^{\mathrm{e}}$ millénaire avant notre ère. 1 : Roc'h Toul, Guiclan, Finistère; 2 : Pont-Glas, Plounéour-Ménez, Finistère; 3 : Kerbizien, Huelgoat, Finistère; 4 : Le Rocher de l'Impératrice, Plougastel-Daoulas, Finistère; 5 : Roc-en-Pail, Chalonnes, Maineet-Loire; 6 : Grainfollet, Saint-Suliac, Ille-et-Vilaine; 7 : Gaéréva, Bréhat, Côtes-d'Armor; 8 : Ménez Dregan, Plouhinec, Finistère; 9 : grottes de 1'Erve dont Rochefort et Margot; Thorigné-en-Charnie, Mayenne; 10 : Saint-Colomban, Carnac, Morbihan; 11 : la Jupinerie, Omonville-la-Petite, Manche; 12 : les Roches du Diable, Locunolé, Finistère; 13 : abri du Rozel, Le Rozel, Manche (DAO G. Marchand).

Fig. 1 - Location of the sites mentioned in the text (grey point) and of the studied sites (black square). The bathymetric curve of - $50 \mathrm{~m}$ indicates the Tardiglacial coastline. The -20 m curve corresponds to the 8th millennium shore. (CAD G. Marchand). 
la Manche, qui semble le plus emblématique. Un massif sableux est plaqué sur la paléofalaise marine et il a préservé deux niveaux d'occupation moustérien marqués par des restes lithiques et des ossements. Le site a fait l'objet de sondages à la fin des années 1960 sous la responsabilité de F. Scuvée et il a été réétudié plus récemment dans une démarche pluridisciplinaire (Van Viel et al., 2006).

Ces quelques cavités creusées par l'océan ne représentent qu'un faible écho du réel potentiel du Massif armoricain, dont les falaises rocheuses recèlent tant de grottes et d'abris dont les remplissages ont été vidés au cours des transgressions marines successives. Les cavités aujourd'hui immergées en permanence représentent également un potentiel à révéler, peut-être moins altéré par le ressac lorsque la remontée des océans fut rapide.

Les abris-sous-roche du domaine continental ont été investis dans une tout autre dynamique de recherche. On a vu plus haut l'importance de la grotte de Roc'h Toul dans les balbutiements des recherches préhistoriques de Bretagne. Il a pourtant fallu attendre le milieu des années 1980 pour voir redémarrer les travaux de terrain sur ces gisements, avec les abris en chaos granitiques du centre Bretagne, occupés au Mésolithique, à Kerbizien, Huelgoat, Finistère (Moullec, 1984 et 1985) et à PontGlas, Plounéour-Menez, Finistère (Le Goffic, 1990). Mais ces cavités n'ont pas été valorisées à leur juste mesure dans les synthèses de l'époque, d'une part car elles ne pouvaient participer à la construction du cadre chronoculturel du Mésolithique à cause de leur faible puissance sédimentaire, d'autre part car elles intervenaient de manière marginale dans les réflexions sur les territoires mésolithiques explorés à travers des centaines de gisements de plein air (Gouletquer et al., 1996).

L'intérêt porté à ces cavités depuis 2007 ne tient pas tant à leur statut de piège sédimentaire qu'à leur spécificité fonctionnelle. Toujours de petites dimensions, elles étaient susceptibles de livrer des types d'occupation complémentaires des grands habitats de plein air. En 2007 et en 2008, la fouille exhaustive du petit abri sous bloc granitique de Pont-Glas a permis d'identifier des occupations mésolithiques aux caractères fonctionnels très particuliers (Marchand et Le Goffic, 2009; Marchand et al., 2011). En 2011, l'abri de Kerbizien a fait l'objet d'une fouille exhaustive, hélas limitée puisque l'essentiel de son remplissage archéologique avait été partiellement détruit en 1983 (Marchand et al., 2014). Les multiples occupations mésolithiques n'ont donc pu y être documentées en place; en revanche, un niveau résiduel de l'Azilien a livré un assemblage lithique original par sa structure fonctionnelle. Elle trouvera peu après des échos dans l'abrisous-roche azilien du Rocher de l'Impératrice (PlougastelDaoulas, Finistère), découvert en 1987 par M. Le Goffic à la faveur d'une tempête et en cours de fouille depuis 2013 (Naudinot, 2013b et 2014). Au contraire de Pont-Glas et Kerbizien qui étaient de petites cavités au sein de boules granitiques, ce site est un abri de modeste superficie au pied d'une immense falaise de quartzite (formation des quartzites de la Roche Maurice, Areginien) qui surplombe la vallée de l'Elorn, au fond de la rade de Brest.
Il paraît nécessaire désormais d'établir un bilan des connaissances sur les quatre sites en grotte et abris occupés au Paléolithique final et au Mésolithique déjà explorés (Roc'h Toul, Pont-Glas, Kerbizien et le Rocher de l'Impératrice), pour comprendre la nature des données archéologiques et ce qu'elles peuvent nous restituer des organisations paléo-économiques.

\section{PREMIERS RÉSULTATS DES FOUILLES DE CAVITÉS ARMORICAINES OCCUPÉES AU PALÉOLITHIQUE FINAL ET AU MÉSOLITHIQUE}

\section{Roc'h Toul à Guiclan}

La grotte de Roc'h Toul à Guiclan (Finistère) bénéficie d'une faille dans un gros bloc de quartzite gris (âge indifférencié Ordovicien-Silurien) avoisinant les $150 \mathrm{~m}$ de haut, qui surplombe la rive gauche de la Penzé (fig. 2). La cavité est formée de deux chambres successives séparées par deux rochers; la première mesure $12,40 \mathrm{~m}$ de long, la seconde $34 \mathrm{~m}$. La hauteur de voute est d'environ $8 \mathrm{~m}$. S'il est difficile de décrire avec précision l'extension de la fouille de M. Le Hir du fait de son ancienneté, il semblerait que ces travaux de vidage des sédiments aient investi la quasi-totalité des deux chambres de la caverne (soit une longueur de près de $50 \mathrm{~m}$ pour une largeur ne dépassant pas $2 \mathrm{~m}$ ).

Du fait de ces méthodes très expéditives visant à retirer « ... tout le sol de la chambre antérieure qui fut répandu en dehors sur la plate-forme »(Le Hir, 1874, p. 88), nous ne disposons que de très peu d'informations sur la stratigraphie du site. Le sol était « ... formé de pierres anguleuses de grès, mélangées à du schiste d'un blanc jaunâtre, et à du quartz, le tout lié par de la terre noire ou jaune, et contenant une grande quantité de charbon végétal. » (Le Hir, 1874, p. 86). Le matériel semble avoir été récolté depuis la surface jusqu'à une profondeur de $50 \mathrm{~cm}$ en relevant « le sol par grandes mottes » (Le Hir, 1874, p. 86). Cette absence de données quant à la stratigraphie du site rend particulièrement difficile la compréhension de cet assemblage. S'agit-il d'un palimpseste? Dans ce cas à quel(s) technocomplexe(s) doivent être rapportées les différentes phases d'installation?

Les premières analyses de l'industrie lithique par M. de Mortillet avait rattaché l'assemblage de Roc'h Toul à « l'âge du renne » (Le Hir, 1869), attribution confirmée plus tard par P. du Chatellier (1888) et J. Déchelette (1908). Sur la foi du faible pourcentage de grattoirs et de burins et de l'importance des denticulés, G. Laplace-Jauretche, rajeunit ensuite la série de plusieurs millénaires pour la placer dans le « complexe arudien » (Laplace-Jauretche, 1957). J.-G. Rozoy (1978) attribua ensuite cette occupation à l'Épipaléolithique ou au Néolithique (mais sans consulter la série). C'est J.-L. Monnier qui, pour la première fois, attribuera le matériel de Roc'h Toul aux « industries aziloïdes » et le placera dans l'Allerød (Monnier, 1980). Les 




Fig. 2 - Entrée de la caverne de Roc'h Toul à Guiclan, Finistère, en 2011 (cliché G. Marchand).

Fig. 2 - Entrance of the Roc'h Toul cave, Guiclan, Finistère in 2011 (photograph G. Marchand).

dernières analyses du matériel ont confirmé cette attribution tout en la restreignant à la phase initiale de l'Azilien (Marchand et al., 2004; Naudinot, 2010).

Si la majorité de ce matériel peut indubitablement être rattaché à cette phase initiale, la présence de plusieurs monopointes à dos courbe (dont plusieurs pointes de Grundy) pose la question d'une éventuelle réoccupation de l'abri par des groupes de l'Azilien récent (fig. 3). Nous verrons plus loin que cette cohabitation des formes bipointes-monopointes est cependant récurrente dans l'Ouest mais également dans d'autres secteurs de l'aire d'extension du techno-complexe azilien. Est-elle révélatrice de mélanges, de l'apparition progressive d'une nouvelle forme de projectile au cours de l'Azilien ancien ou encore d'une variabilité typologique à mettre en relation avec la disponibilité en matériaux lithiques?

L'industrie lithique de Roc'h Toul est essentiellement réalisée sur du silex, probablement originaire des cordons littoraux du Nord du Finistère (fig. 3). Quelques pièces ont été débitées dans un silex plus opaque, gris-noir, sans équivalent parmi les galets que l'on peut glaner sur le littoral actuel, mais montrant de grandes similitudes avec les matériaux rencontrés sur la bande jurassique de la marge orientale du Massif armoricain. Il faut noter également la présence de quelques rares pièces extraites dans une calcédoine rouge/orangé. Même si la grande majorité des zones corticales sont roulées et témoignent d'un transport maritime, d'autres semblent moins altérées et certaines ont même un cortex natif. Une grosse part de l'approvisionnement s'est donc à l'évidence déroulée le long du littoral finistérien. Une autre partie cependant a pu être collectée directement sur des affleurements encore exondés de la Manche ou sur des cordons littoraux aujourd'hui inondés proches de ces affleurements. Différents types de microquartzites calcédonieux, parfois très fins, probablement originaires de la région de la Forest-Landerneau à une quarantaine de kilomètres du site, ont également été débités à Roc'h Toul, notamment pour fabriquer une des bipointes de l'assemblage.

Il est particulièrement difficile dans les conditions exposées au-dessus de décrire en détail les objectifs et méthodes de production des occupants de la caverne de Roc'h Toul. Les objectifs du débitage semblent assez diversifiés (s'il ne s'agit pas d'une conséquence de la présence de différentes composantes culturelles parmi l'assemblage collecté dans la grotte). La série est dominée par les supports retouchés, denticulés et pièces encochées mais compte également quelques grattoirs, troncatures et rares burins. À noter la présence de quelques fragments de lames présentant une retouche rasante scalariforme tout à fait caractéristique des premiers temps de l'Azilien. Les petites lames ont été utilisées pour la fabrication des pointes de projectiles. Parmi ces huit éléments on compte deux bipointes entières accompagnées de deux 




Fig. 3 - Armatures aziliennes de Roc'h Toul à Guiclan, Finistère (cliché N. Naudinot).

Fig. 3 - Azilian arrowheads of Roc'h Toul, Guiclan, Finistère (photograph N. Naudinot).

fragments pouvant s'y rattacher, deux pointes de Grundy entières, un fragment apical de (bi-?)pointe à dos courbe, ainsi qu'un élément distal de lamelle à bord abattu. Les bipointes ont été réalisées sur des lames étroites et régulières. Les Préhistoriques ont en revanche plutôt choisi des supports, certes allongés, mais peu calibrés, pour la fabrication des monopointes.

Les éclats, bien que peu représentés, ont été utilisés pour la confection des grattoirs. Les nucléus observés témoignent d'une exploitation laminaire hormis pour un exemplaire multipolaire présentant des négatifs d'extraction de petits éclats et de lamelles irrégulières. Hormis cette pièce un peu particulière qui rappelle certains exemplaires du premier Mésolithique, aucun véritable nucléus à éclats n'a été identifié. Ce sont donc les lames qui semblent avoir été au cœur des intentions du débitage à Roc'h Toul (bien que la part du lamellaire puisse être sous-estimée du fait de l'absence de tamisage fin). Compte tenu des problèmes liés à l'ancienneté des fouilles, il est difficile de déterminer les méthodes de production mises en œuvre à Roc'h Toul par les occupants aziliens. Peu d'indices témoignent de la mise en place de crêtes. Des enlèvements transversaux sont toutefois présents sur un des nucléus et quelques néocrêtes ont été identifiées dans la série. La plupart des supports semblent ainsi avoir été extraits à la pierre. Il est néanmoins difficile de préciser exactement le type de percuteur (tendre ou dur). La pre- mière hypothèse est privilégiée mais l'apparente hétérogénéité de la série ne permet pas de l'affirmer.

Compte tenu de la nature de la fouille de ce gisement et des problèmes de conservation du matériel, dans le meilleur des cas, éparpillé dans différents lieux de conservation, il est malheureusement impossible de proposer une quelconque hypothèse quand aux modalités d'occupation de cette cavité finistérienne au cours de l'Azilien ancien... Une nouvelle opération de fouille de Roc'h Toul, se focalisant sur l'entrée de la grotte et sur les quelques zones non investies par la fouille initiale est ainsi prévue par l'un d'entre nous (N. N.) dans les années à venir.

\section{Pont-Glas à Plounéour-Ménez}

Installé sur un replat très faiblement incliné, l'abri-sousroche de Pont-Glas à Plounéour-Ménez (Finistère) naît du chevauchement de deux blocs granitiques (fig. 4 et 5). L'abri s'ouvre à l'est par un auvent de $2 \mathrm{~m}$ de hauteur à son maximum. Une seconde entrée de $1,40 \mathrm{~m}$ de haut existe aussi à l'ouest. L'abri affecte un plan grossièrement triangulaire de $5 \mathrm{~m}$ de côté (axe nord-sud) et de $6 \mathrm{~m}$ de hauteur (axe est-ouest). La surface protégée par les rochers est d'environ $15 \mathrm{~m}^{2}$, mais l'inclinaison des deux blocs ne rend réellement accessible qu'une aire de $8 \mathrm{~m}^{2}$ et encore fautil composer avec un bloc fiché au centre de l'espace, sur lequel on peut éventuellement s'asseoir. C'est donc sans 


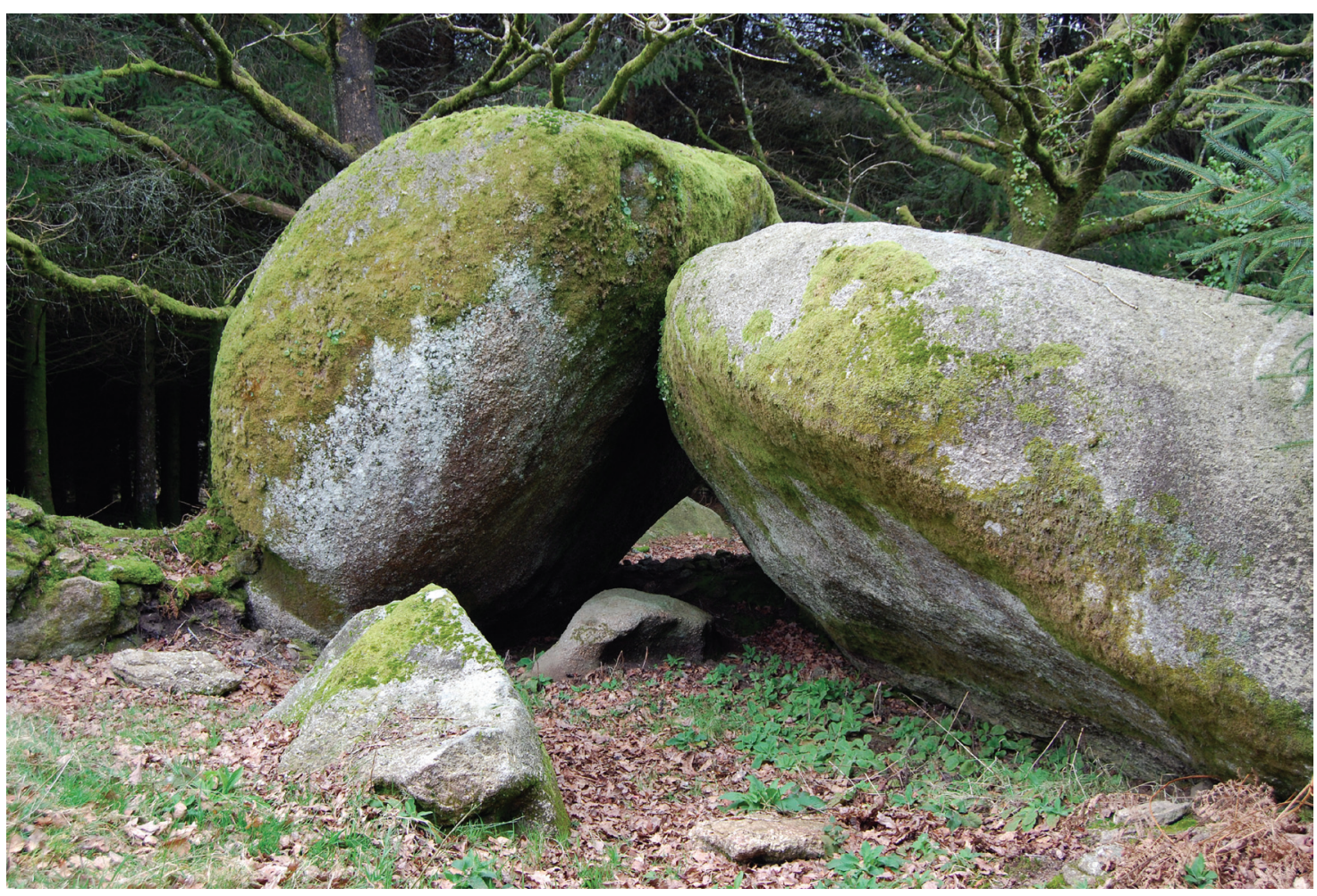

Fig. 4 - L'abri-sous-roche de Pont-Glas, Plounéour-Ménez, Finistère, en 2011. Le débitage du bloc devant à gauche est dû à l'action des carriers à une époque historique (cliché G. Marchand).

Fig. 4 - The Pont-Glas rock-shelter, Plounéour-Ménez, Finistère, in 2011. The granitic block in front on the left was damaged by quarrymen in historical times (photograph G. Marchand).

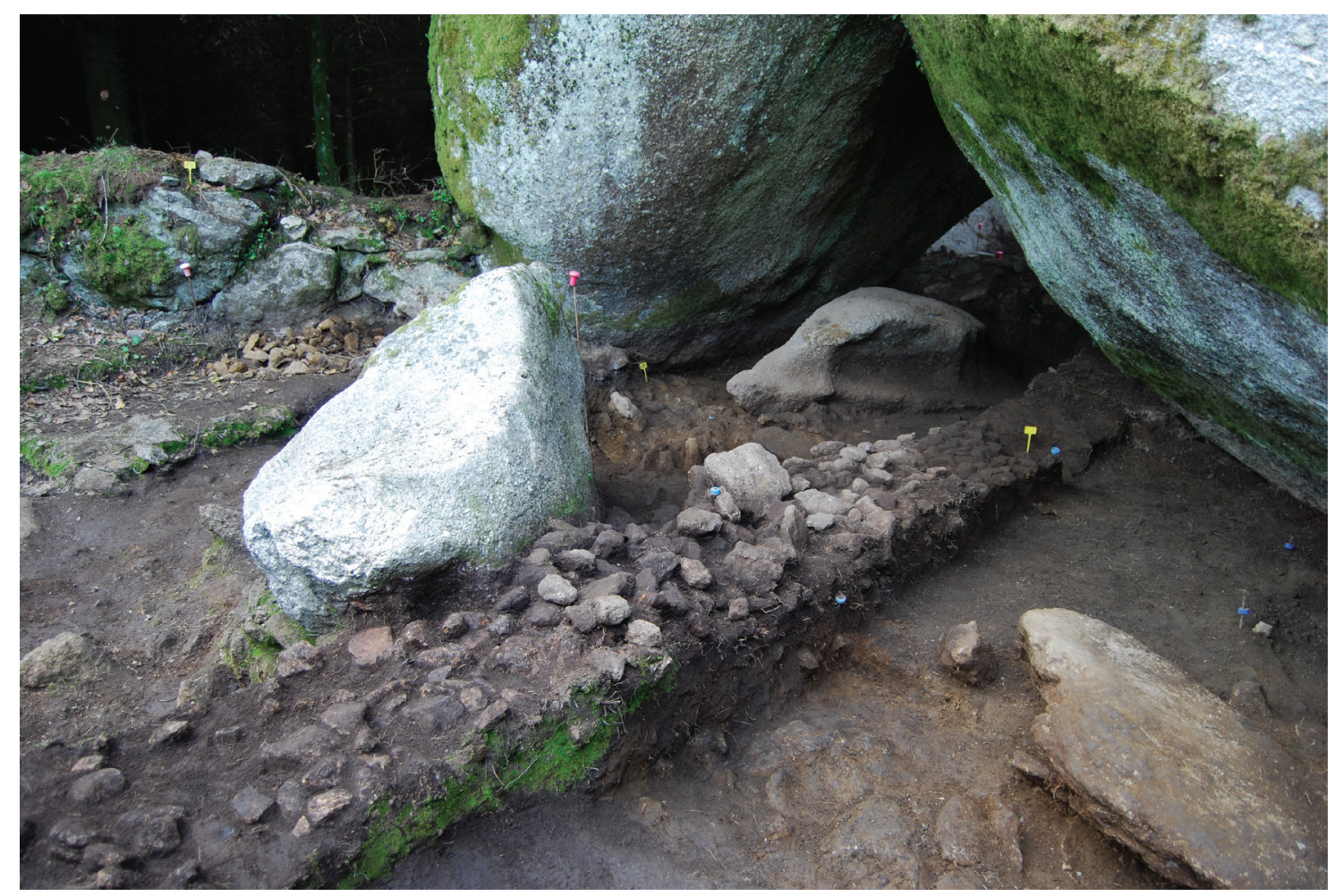

Fig. 5 - Pont-Glas, Plounéour-Ménez, Finistère, en cours de fouille (2008). Les pierres brûlées au sommet de la berme correspondent aux occupations historiques. Le niveau mésolithique est à la base de cette berme, de part et d'autre (cliché G. Marchand).

Fig. 5 - The 2008 excavation at Pont-Glas, Plounéour-Ménez, Finistère. The burned stones at the top of the baulk correspond to historical occupation. Mesolithic level is at the base of the baulk, on either side (photograph G. Marchand). 
conteste la plus petite caverne explorée pour l'instant en Bretagne. Après un premier sondage en 1987 réalisé par M . Le Goffic, son inventeur, une fouille totale de l'abri et de ses abords a concerné environ $40 \mathrm{~m}^{2}$ (Marchand et al., 2011). La stratigraphie de l'abri était relativement simple, avec de haut en bas, sur une épaisseur d'un demi-mètre, une litière sèche et un niveau d'humus, un niveau de très nombreuses pierres chauffées à l'extérieur et dans l'entrée orientale de l'abri, un ensemble de niveaux à matrice arénacée et la roche mère plus ou moins disloquée et arénisée.

Le mobilier lithique mésolithique était présent dans tous les niveaux, ce qui témoigne des nombreuses perturbations du sol par les fouisseurs au cours du temps. Une importante occupation de La Tène ancienne n'est attestée que dans l'US 1 et 2, avec entre autres de la poterie utilitaire et au moins deux beaux vases décorés. L'abri a livré un foyer soigneusement empierré et jointoyé à l'argile, aux bords hélas démembrés, puis une quinzaine de centimètres plus bas un épandage de charbon correspondant probablement à un autre foyer à plat. Au même endroit mais dans une unité stratigraphique arénacée encore plus basse, une zone de piétinement affectait directement le substrat. Cet empilement signale bien évidemment les fortes contraintes naturelles de cet espace de vie.

Avec 998 éléments recueillis en fouille et par tamisage systématique à l'eau, il s'agit d'une très petite série, en regard des immenses sites mésolithiques en Bretagne. Le mobilier lithique comprend une composante ancienne (VIII ${ }^{\mathrm{e}}$ millénaire), avec des triangles scalènes étroits, des lamelles à dos étroites et des pointes à base retouchée.
Un petit pic en microquartzite de la Forest-Landerneau évoque immanquablement les groupes mésolithiques de Basse-Normandie et du Bassin parisien du Mésolithique moyen; c'est le premier outil prismatique découvert en Bretagne. La seconde composante est la plus importante en nombre d'armatures et correspond sans conteste au Mésolithique final, plus particulièrement au Téviecien de faciès Beg-er-Vil où dominent les bitroncatures symétriques (fig. 6). La date de cette dernière dans la seconde moitié du VI ${ }^{\mathrm{e}}$ millénaire avant notre ère est confirmée par une datation sur charbon prise dans l'unité stratigraphique la plus basse $(6400 \pm 50 \mathrm{BP}$, soit de 5480 à 5300 avant notre ère; Beta-253526). L'analyse des chaînes opératoires montre une grande diversité de roches locales taillées au côté des galets de silex, qui restent dominants ( $44 \%$ des pièces). La rareté des phases initiales du débitage est à noter, de même que l'abondance des armatures (67\% des outils aménagés) et aussi le nombre conséquence de fractures à l'impact (13 sur 42), tout particulièrement sur les trapèzes symétriques emmanchés en flèche tranchante. Ces caractères fonctionnels forts se distinguent avant tout sur la composante récente.

L'analyse spatiale des vestiges lithiques révèle quelques lignes de force intéressantes, malgré la très forte perturbation des lieux par les hommes et les animaux fouisseurs. On constate ainsi la concentration des pièces lithiques sous le bloc 2, avec un décalage horizontal partiel entre les composantes ancienne et récente du Mésolithique. Cette distinction est également perceptible contre toute attente dans la dimension verticale, avec évidemment



Fig. 6 - Pont-Glas, Plounéour-Ménez, Finistère. Bitroncature symétrique en silex avec deux traces d'impact burinantes liées à un usage en flèche tranchante; longueur du tranchant : $12 \mathrm{~mm}$ (cliché G. Marchand).

Fig. 6 - Pont-Glas, Plounéour-Ménez, Finistère. ). Symmetrical trapeze in flint with two burin-like impacts linked to use as a transverse arrowhead (photograph G. Marchand). 
un fort brouillage lié aux remaniements. Ainsi la composante ancienne (pointes à base concave et triangles scalènes) se trouve en quasi-totalité dans les unités stratigraphiques arénacées inférieures, tandis que les trapèzes de la composante récente reposent principalement dans les unités stratigraphiques supérieures limoneuses, très perturbées.

Il faut signaler que des sondages ont été réalisés dans trois massifs granitiques sur les pentes environnant PontGlas, sans résultats archéologiques probants. On notera seulement que les trois sondages menés dans le vaste chaos rocheux de Viou Euzc'hlazard ont montré des lessivages récents entre les boules géantes, un processus très contraignant qui évidemment va être un frein aux recherches dans ces contextes.

\section{Kerbizien à Huelgoat}

L'abri-sous-roche de Kerbizien est inclus dans un des nombreux blocs granitiques de la partie orientale du massif de granite de Huelgoat, sur la commune du même nom, au centre du Finistère. Ouvert vers l'ouest en partie amont d'un petit thalweg très pentu, en sommet de coteau, l'abri de Kerbizien est particulièrement impressionnant par son aspect monolithique (Marchand et al., 2014). La cavité est creusée aux dépens d'un bloc de granite haut de $6,77 \mathrm{~m}$ depuis le sol actuel, long de 19,40 m (axe nord-sud) et large de 18,20 m (axe est-ouest). Elle mesure 9,50 $\mathrm{m}$ à l'ouverture pour environ $5,50 \mathrm{~m}$ de profondeur, avec une hauteur sous plafond de 2,80 m depuis le socle rocheux et une surface habitable d'environ $11 \mathrm{~m}^{2}$ (fig. 7).

Alors qu'il était intégré dans un aménagement paysager à proximité d'une maison individuelle, son remplissage sédimentaire a été presque intégralement détruit au début des années 1980. Les lignes encore visibles sur les parois dénoncent une épaisseur d'environ un mètre dans toute la cavité. J.-M. Moullec avait alors conduit un tamisage des déblais et un sondage dans la cavité, livrant 2940 pièces d'après des rapports inédits (Moullec, 1984 et 1985). Elles avaient permis de décrire avec précision les différentes composantes chronoculturelles présentes : le Paléolithique final (Azilien), sous la forme de très nombreuses pointes aziliennes, de grattoirs sur bout de lame et de débitage laminaire à partir de deux plans de frappe opposés; le premier Mésolithique (groupe de Bertheaume, daté aujourd'hui de l'intervalle 8200-7500 avant notre ère), avec des triangles scalènes étroits et de nombreuses lamelles à dos étroites,; le second Mésolithique (Téviecien, daté de la fin du $\mathrm{VII}^{\mathrm{e}}$ et du $\mathrm{VI}^{\mathrm{e}}$ millénaire avant notre ère), plus discret mais marqué par les trapèzes symétriques; une période historique, pendant laquelle l'habitat avait servi d'atelier métallurgique (nombreuses scories et tessons).

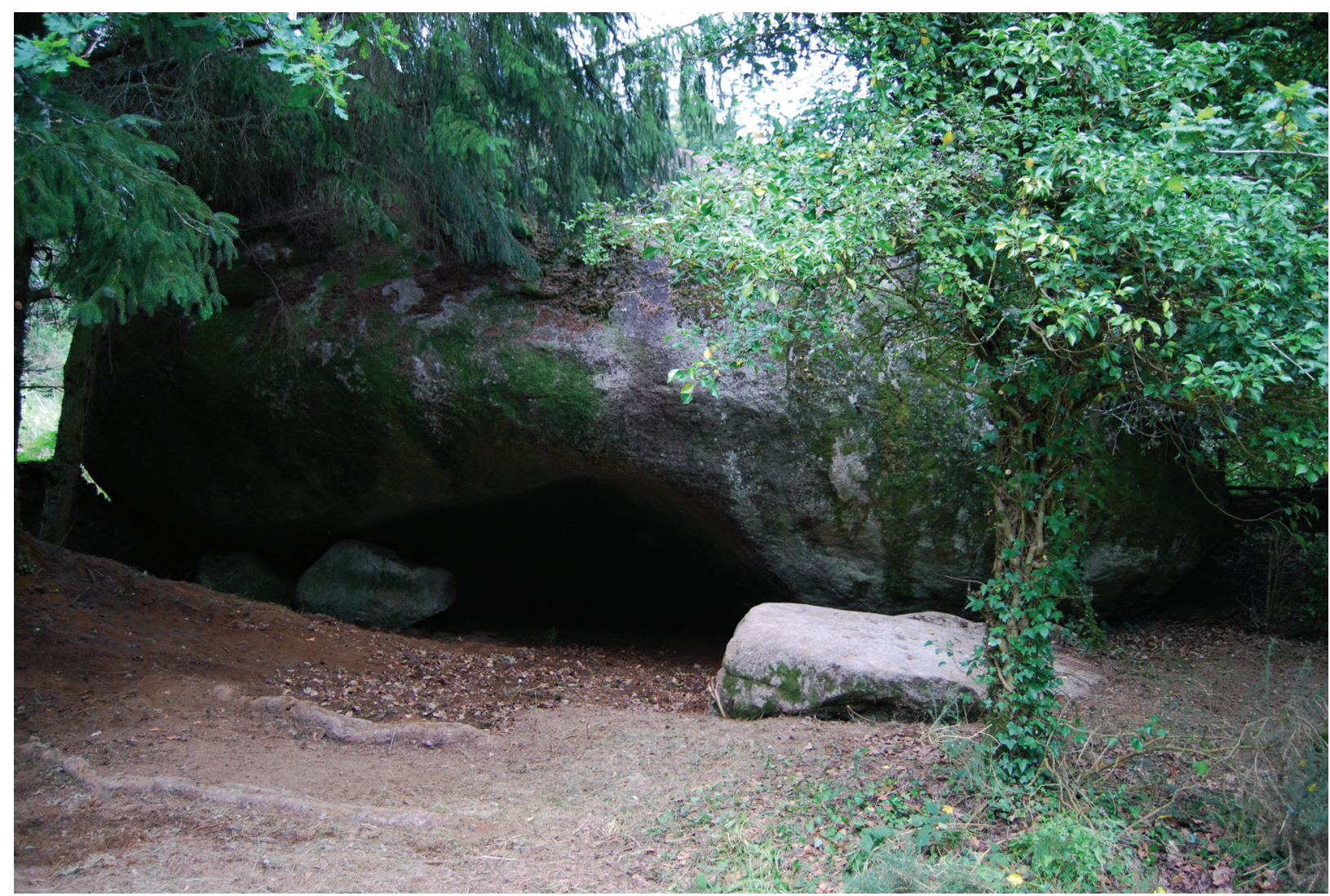

Fig. 7 - Kerbizien, Huelgoat, Finistère. Le site après le rebouchage de la fouille en 2011. Le bloc devant l'abri a été débité dans sa partie gauche probablement à une époque historique (cliché G. Marchand).

Fig. 7 - The Kerbizien rock-shelter, Huelgoat, Finistère, after the 2011 excavation. TThe left part of granitic block at the entrance was probably damaged by quarrying during historical times (photograph G. Marchand). 
La fouille réalisée pendant deux semaines en juillet 2011 a permis seulement d'identifier des niveaux en place $\mathrm{du}$ Tardiglaciaire au fond de la caverne et en partie sud du bloc (fig. 8). Les niveaux résiduels en place formaient une bande de deux mètres de large le long de la paroi. L'industrie lithique y a été découverte dans deux unités stratigraphiques superposées, constituée de limons exogènes et $\mathrm{d}$ 'arène granitique issue des parois, sur une épaisseur maximale de $15 \mathrm{~cm}$. Des limons lœssiques datant probablement du Pléniglaciaire nappent l'avant de l'abri, mais ils n'ont enregistré aucune occupation du Paléolithique et ils ont été décapités par l'action d'un bulldozer. La totalité du site a été fouillée, soit environ $8 \mathrm{~m}^{2}$ d'Azilien en place dans la cavité. L'inclinaison de la paroi n'en fait pas une aire d'habitat; il ne s'agit pas non plus d'une zone de déchets préférentielle, car nous observons de faibles densités, comprises entre 8 et 26 pièces au $\mathrm{m}^{2}$. Vers l'extérieur, à l'emplacement d'une zone de gouttière, cette densité (esquilles comprises) atteint 86 pièces au $\mathrm{m}^{2}$, ce qui reste faible.

Parmi les 1313 objets archéologiques issus de la fouille et du tamisage, $20 \%$ proviennent des unités stratigraphiques tardiglaciaires en place. Il s'agit principalement de silex, mais aussi de grès éocène, de microquartzite, de cataclasite et de calcédoine. Ils témoignent d'échanges ou de parcours à des distances d'une cinquantaine de kilomètres au nord vers les rivages de la Manche; pour de plus rares silex, les niveaux jurassiques ou crétacés à l'est du Massif armoricain forment un autre pôle d'approvisionnement, qui invitent à imaginer des déplacements de plus de $300 \mathrm{~km}$. Dans ces niveaux aziliens résiduels, on a recueilli également de petites plaquettes de schiste (cinq éléments de plus de $51 \mathrm{~mm}$, 51 éléments d'une longueur comprise entre 11 et $50 \mathrm{~mm}$ et 110 fragments d'une taille inférieure à $10 \mathrm{~mm}$ ), sur lesquelles aucune gravure n'a été identifiée. Leur origine se trouve au minimum à $4 \mathrm{~km}$ de Kerbizien.

Les pièces lithiques sont peu affectées par le feu $(7,5 \%$ pour les seuls silex). Les produits lamino-lamellaires y sont en forte proportions avec $22,6 \%$ de l'ensemble des objets bruts et retouchés toutes roches confondues, ou $24,3 \%$ pour les seuls silex. Les chaines opératoires de production sont très incomplètes. Il y a clairement une grande variété de standards de production, de la lamelle à la lame large, depuis des largeurs de $5 \mathrm{~mm}$ jusqu'à celles de $24 \mathrm{~mm}$. Les pointes aziliennes (monopointes et bipointes, entières ou fragmentées) sont au nombre de vingt et un et montrent une très grande variété de modules et de matériaux (fig. 9). Les lames longues de plus de $80 \mathrm{~mm}$ et larges de 20 à $25 \mathrm{~mm}$ sont un autre des objectifs

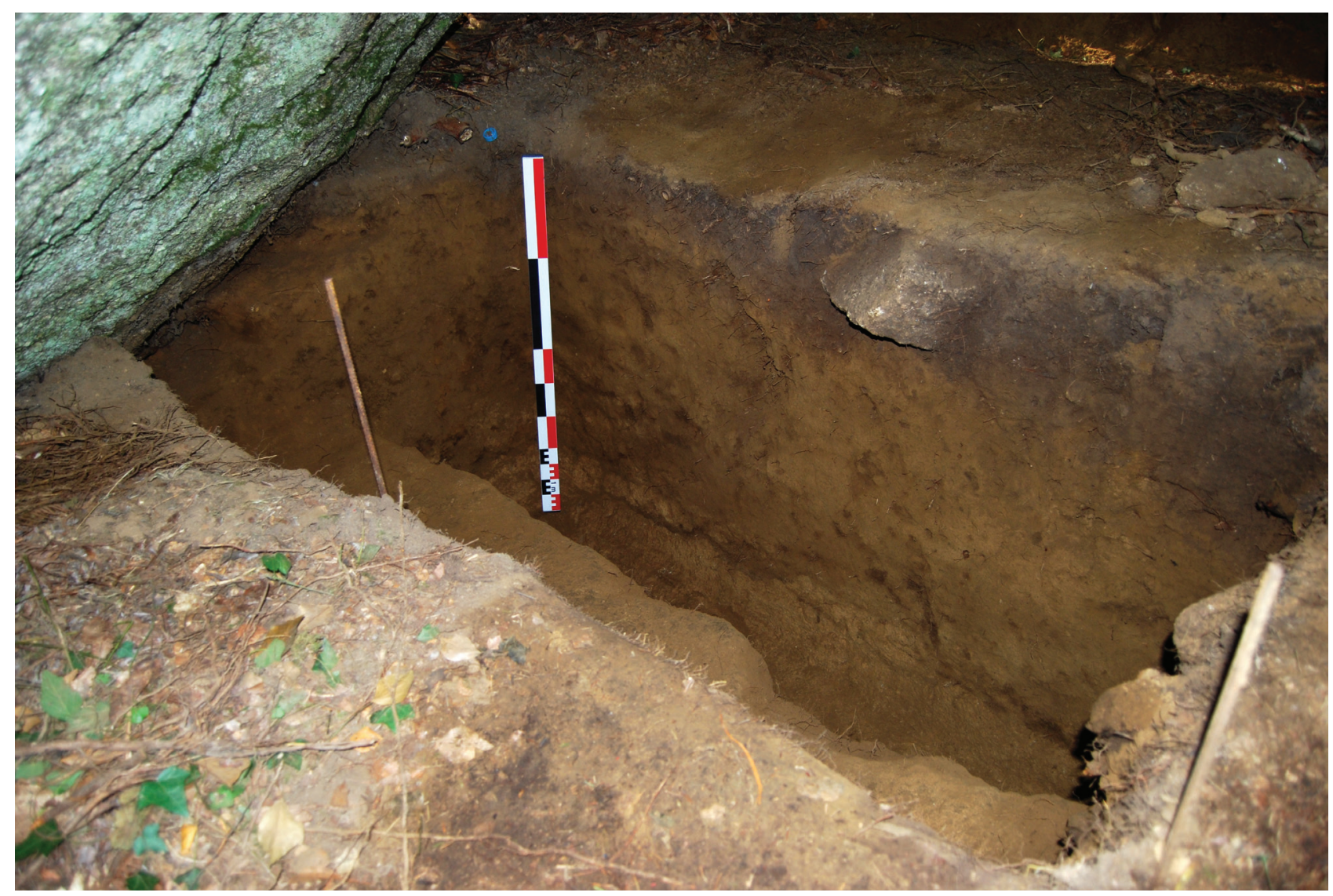

Fig. 8 - Kerbizien (Huelgoat, Finistère), fouille 2011. Au sud-ouest du rocher, le petit surplomb protège un dépôt de lœss épais de 1,20 m. Les industries lithiques aziliennes et mésolithiques étaient ici mêlées dans le sol actuel en sommet de coupe (cliché G. Marchand).

Fig. 8 - The Kerbizien rock-shelter (Huelgoat, Finistère) during the 2011 excavation. Southwest of the rock, the small overhang protects a loess deposit $1.20 \mathrm{~m}$ thick. The Azilian and Mesolithic lithic industries were mixed here in the modern soil at the top of the section (photograph G. Marchand). 
de débitage très marquants, d'autant que certains exemplaires ont été conservés entiers sous la forme de couteaux aux bords très retouchés ou ébréchés. Enfin les chutes de burin, souvent très régulières et soigneusement préparées, sont nombreuses, alors qu'un seul burin a pu être exhumé.

Le spectre fonctionnel de l'industrie azilienne est donc fort singulier : de grandes lames usagées, des pointes aziliennes dans divers états et des nombreuses chutes de burin. Les unités stratigraphiques de Kerbizien plus hétérogènes fouillées en 2011 ne viennent pas transformer notablement ce spectre fonctionnel, pour autant que l'on puisse en juger. L'ensemble permet d'évoquer un Azilien ancien. La signature fonctionnelle de cet assemblage évidemment amputé par les destructions serait donc tout à fait compatible avec l'hypothèse de stations logistiques (c.-à-d. de haltes de chasse) de groupes très mobiles. Le mobilier lithique du Mésolithique est très abondant, mais il n'a pas été découvert en place et il comprend plusieurs périodes. Il serait aberrant d'en définir les caractères fonctionnels, mais rien n'incline à penser que l'on ait eu affaire - comme à Pont-Glas - à des stations logistiques.

\section{Le Rocher de l'Impératrice à Plougastel-Daoulas}

L'opération archéologique en abri la plus récente, toujours en cours, a eu lieu à Plougastel-Daoulas dans le Finistère, sur le site du Rocher de l'Impératrice (Naudinot, 2013b et 2014). Les résultats présentés ici sont ainsi tout à fait préliminaires et seront développés plus en détail dans une prochaine publication.

Cette cavité est localisée au pied d'une immense falaise de quartzite d'une quarantaine de mètres de haut (fig. 10), exposée au nord-ouest et dominant d'une centaine de mètres le fond de la rade de Brest et l'estuaire du fleuve Élorn. Avec sa dizaine de mètres de long et ses trois mètres de profondeur maximum, l'abri du Rocher de l'Impératrice est pourtant modeste (fig. 11). Il est prolongé quelques mètres au sud par un surplomb couvrant une zone d'une petite dizaine de mètres carrés constituant un second abri.

Le site a été découvert par M. Le Goffic alors conservateur de l'Archéologie du département du Finistère après des creusements réalisés par des escaladeurs pour

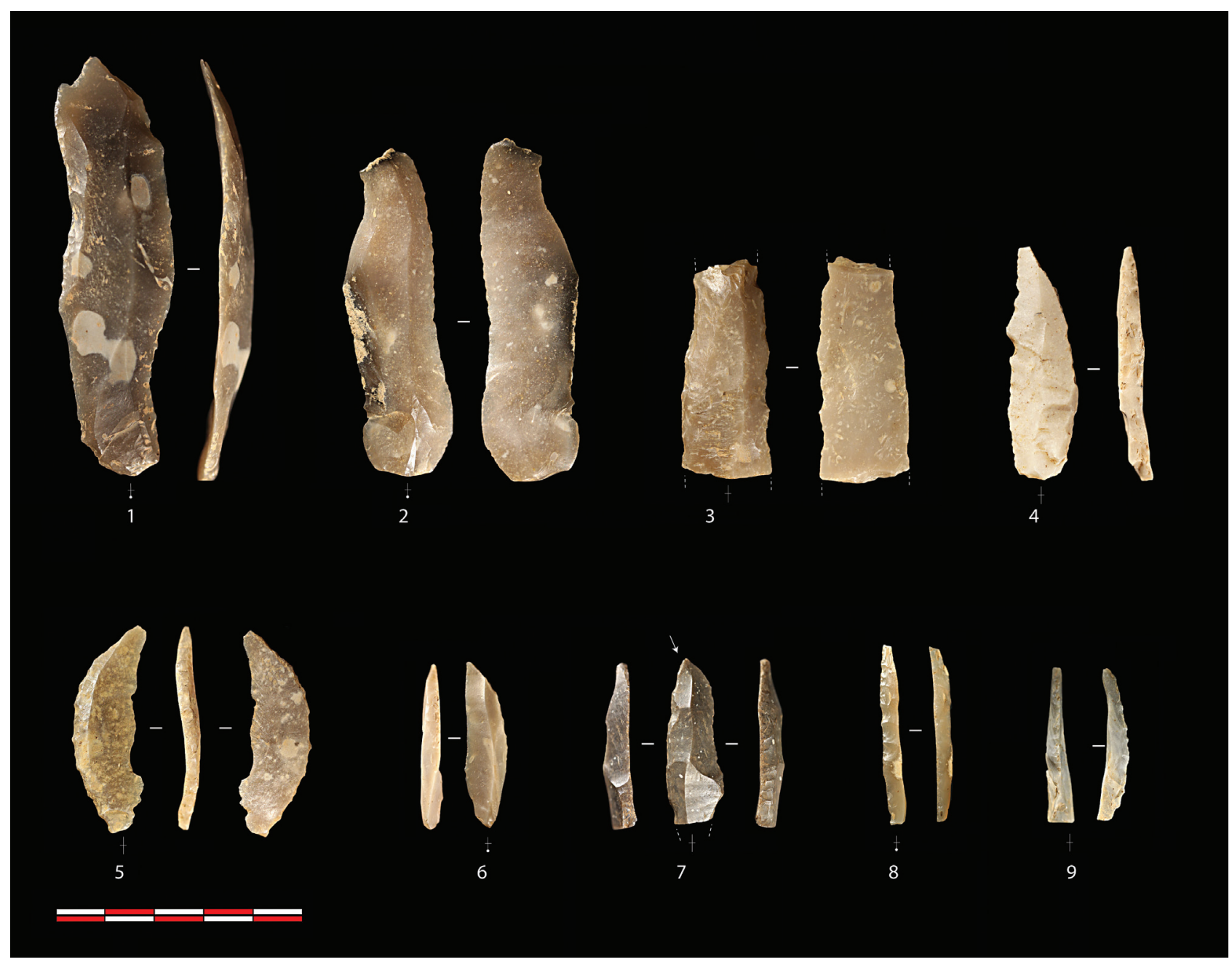

Fig. 9 - Kerbizien (Huelgoat, Finistère). Industrie en silex des niveaux aziliens. 1-3: lames utilisées; 4-7 : pointes à dos courbe; 8-9 : chutes de burin (clichés N. Naudinot).

Fig. 9 - Kerbizien (Huelgoat, Finistère). Flint industry from the Azilian levels. 1-3: utilized blades; 4-7: curved back points; 8-9: burin spalls (picture N. Naudinot). 


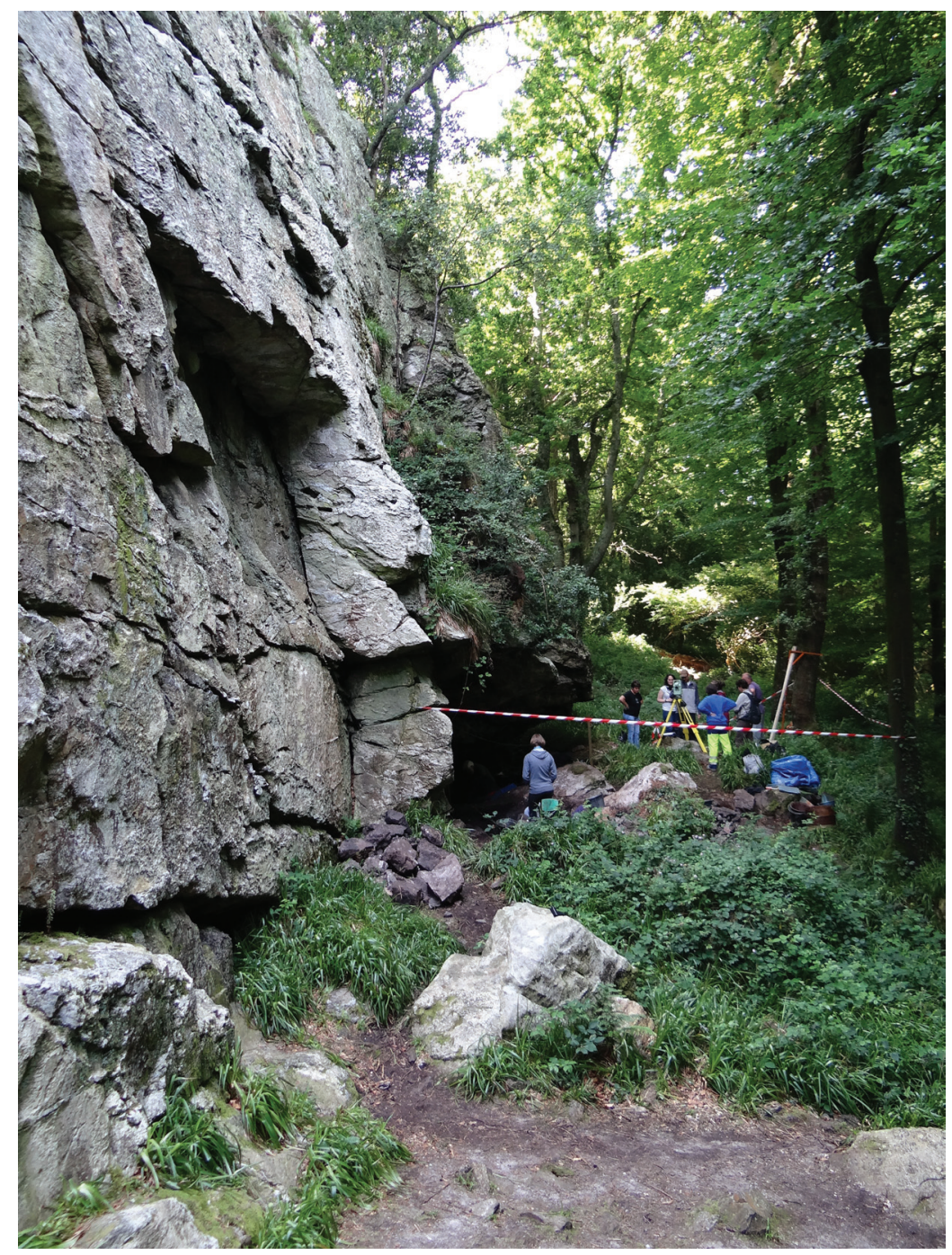

Fig. 10 - Le Rocher de l'Impératrice, Plougastel-Daoulas, Finistère, en cours de fouille en 2013, vu de l'est (cliché G. Marchand).

Fig. 10 - Le Rocher de l'Impératrice, Plougastel-Daoulas, Finistère, during the 2013 excavation seen from the east (photograph G. Marchand).

augmenter le surplomb de l'abri. Une approche initiale de cette petite série a permis en 2008 de se faire une première idée de la composition de l'assemblage lithique et surtout de révéler le très important potentiel archéologique de ce gisement (Naudinot, 2010). Le terrain a récemment été acheté par le conseil général du Finistère, notamment afin de protéger une flore rare (dont Hymenophyllum tunbrigense et Dryopteris aemula). Cette acquisition a permis en juillet 2013, 26 ans après la découverte du site, de lancer une première campagne de sondage en 2013 puis en fouille, toujours en cours depuis 2014.

Les premières campagnes, qui ont investi une trentaine de mètres carrés, n'ont pas encore permis d'atteindre le substrat et la stratigraphie de l'abri reste donc encore partielle. Le remplissage est riche en blocs d'effondrement. Certaines de ces roches dépassent aisément les dix tonnes. S'il est trop tôt pour décrire précisément la stratigraphie complète de l'abri, on notera la présence à la base de la zone actuellement fouillée d'une couche sédimentaire très fine jaunâtre à la surface de laquelle la plupart du matériel a été mis au jour. Ce dépôt, s'apparentant fortement à du lœss, est très induré par la présence d'oxydes ferromanganiques. Plusieurs secteurs du site ont été profondément perturbés par différents agents naturels et anthropiques. En dehors des classiques effets de parois et terriers, des creusements parsemés ont porté atteinte à l'intégrité du site. Ces perturbations sont à la fois le fait des aménagements des escaladeurs à l'origine de la découverte du gisement, d'aménagement du site par les troupes de la Wehrmacht lors de la seconde guerre mondiale, mais également de fouilles clandestines. Ces pillages anciens ne semblent néanmoins pas avoir eu pour cible les vestiges préhistoriques. Le site semble plutôt avoir souffert d'une légende, ayant donné son nom au site, selon laquelle, lors de sa visite à Plougastel-Daoulas en compagnie de l'empereur Napoléon III, l'impératrice Eugénie aurait fait tomber une de ses bagues du haut de la falaise à la hauteur de l'abri-sous-roche... Malgré ces perturbations, l'étude de la répartition des remontages-raccords, les projections du matériel, ainsi que les premiers 




Fig. 11 - Le Rocher de l'Impératrice (Plougastel-Daoulas, Finistère). Vue de détail de l'abri (cliché N. Naudinot).

Fig. 11 - Detail view of the rock-shelter of Le Rocher de l'Impératrice, Plougastel-Daoulas, Finistère (photograph N. Naudinot).

résultats de l'analyse géomorphologique, semblent bien suggérer la présence de niveaux aziliens en place sur le site. Les prochaines campagnes de fouilles, tout comme les études sédimentologiques et microstratigraphiques en cours, apporteront plus d'informations sur ces questions.

Le matériel recueilli apparait comme homogène. Comme les pièces découvertes en surface par M. Le Goffic, ils plaident pour une occupation de l'abri durant la phase ancienne de l'Azilien. Quelques éléments évoquent toutefois des occupations plus récentes de l'abri au cours du Mésolithique, du Néolithique et à la Protohistoire. La série pour le moment recueillie est composée de 460 pièces enregistrées en trois dimensions auxquelles s'ajoutent 558 pièces collectées au tamisage en 2013 et un nombre encore indéterminé (encore en cours de tri) de vestiges récoltés de la même manière lors de la dernière campagne de 2014. Cette industrie est très essentiellement débitée dans du silex. Si l'origine marine de certains rognons est évidente, une part des supports, par la présence de cortex mais également par les dimensions des lames, semble suggérer un approvisionnement en partie réalisé directement sur affleurements crétacés de la Manche ou dans des paléocordons littoraux plus proches de ces affleurements et aujourd'hui submergés. À noter la présence de quelques éléments en silex d'origine plus clairement allochtone. Il s'agit d'un fragment mésial de lame large dans un matériau gris-noir de type packstone très riche en éléments détritiques rappelant le silex, disponible dans les formations du Bajocien de la région de Sablé-sur-Sarthe sur les marges jurassiques du Massif armoricain, ainsi que d'une lame en silex dit « grain de mil », probablement originaire de Charente-Maritime. Ces pièces très caractéristiques suggèrent des circulations sur des distances dépassant les $400 \mathrm{~km}$. Environ 3\% des pièces lithiques ont également été débités dans un microquartzite calcédonieux probablement originaire du secteur de la Forest-Landerneau, à quelques kilomètres au nord-est du site.

C'est l'obtention de lames qui structure les schémas opératoires de production lithique au Rocher de l'Impératrice. Les supports utilisés bruts ou transformés en pointes de projectile ou outils retouchés sont en effet dans près de $90 \%$ des cas des lames. Les lames les plus larges semblent avoir été utilisées brutes comme le suggèrent les nombreuses ébréchures sur leurs tranchants. Elles ont également été retouchées, notamment selon une modalité chère aux groupes de l'Azilien ancien, avec une retouche rasante/semi abrupte scalariforme tout à fait caractéris- 
tique. Ces lames ont également été transformées en grattoirs, burins et troncatures. Les petites lames ont quant à elles été utilisées afin de concevoir les nombreuses pointes de projectiles du site (fig. 12). Ces armatures sont essentiellement représentées par des bipointes à dos courbe qui, comme dans le niveau 4 du Bois-Ragot (Plisson, 2005), semblent avoir été emmanchées légèrement désaxées si l'on en croit l'orientation des stigmates d'impacts et de micro-polis linéaires. Comme pour la grotte de Roc'h Toul et l'abri de Kerbizien, mais également comme la plupart des rares sites aujourd'hui connus pour l'Azilien ancien, la série contient également quelques exemples de monopointes à dos courbe dont trois éléments de pointes de Grundy. Le rattachement d'une partie de ces mono-

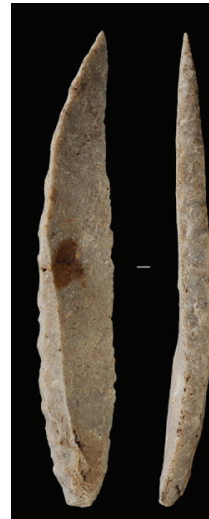

1

10



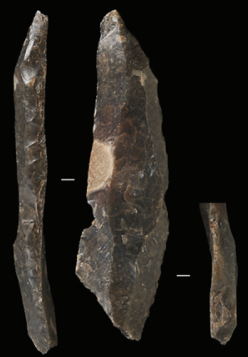

2

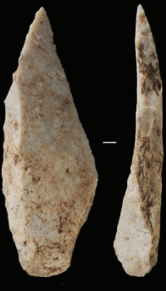

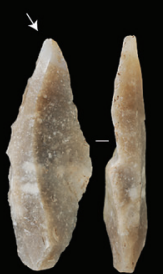

4

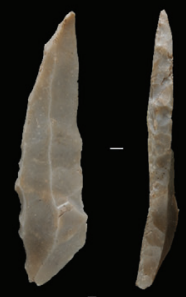

5

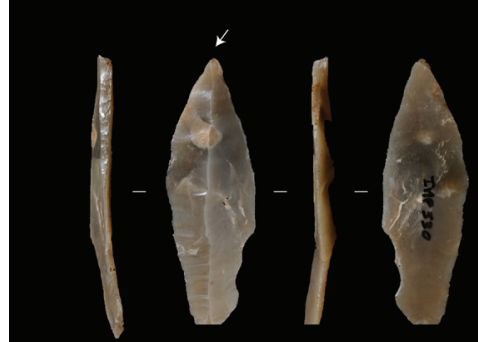

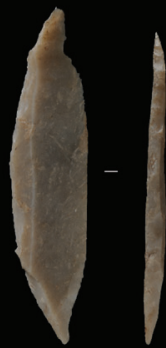

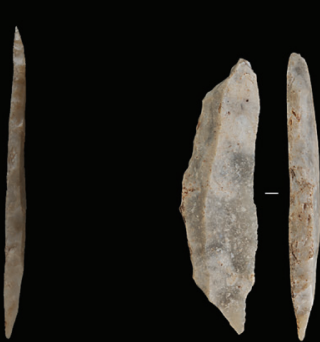

12

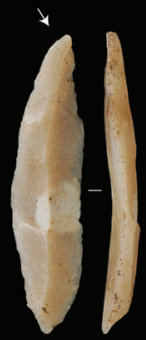

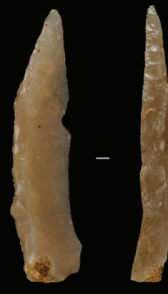

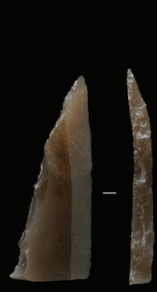

15



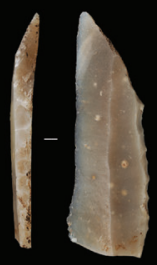



Fig. 12 - Le Rocher de l'Impératrice, Plougastel-Daoulas, Finistère. Pointes à dos courbe (cliché N. Naudinot).

Fig. 12 - Le Rocher de l'Impératrice, Plougastel-Daoulas, Finistère. Curved back points (picture N. Naudinot). 
pointes à la composante Azilien ancien est possible du fait de la nature des supports investis et du soin apporté à la retouche. Pour d'autres pièces, aménagées sur des supports épais et irréguliers, plusieurs hypothèses restent envisageables pour expliquer leur présence dans la série : palimpseste de plusieurs occupations aziliennes s'étalant tout au long de la période, développement de la forme bipointe au cours de l'Azilien ancien, ou, scénario privilégié pour le moment, fabrication de monopointes sur des supports moins réguliers ne permettant pas la conception de bipointes dans l'optique d'une gestion particulière des matériaux lithiques. La poursuite de l'opération permettra certainement d'avancer sur cette question.

La fouille de ce nouveau gisement de référence pour l'Azilien de l'Ouest de la France a également permis la découverte de témoignages particulièrement singuliers dans le grand Ouest, mais également rares pour cette période au niveau européen, sous la forme d'une vingtaine d'éléments de plaquettes de schiste gravées, portant des tracés abstraits, mais également des figurations animalières, et présentant des traces de colorant noir dans les gravures. Ces éléments, toujours en cours d'étude, sont particulièrement intéressants dans l'optique d'une réflexion sur la transformation des registres symboliques au cours du Paléolithique final et feront l'objet de publications plus détaillées dans les mois à venir.

Les résultats de cette nouvelle campagne de fouille au Rocher de l'Impératrice apportent des informations préliminaires quant au statut socioéconomique du site. Il est toutefois encore trop tôt pour conclure sur ces questions, qui continueront d'être au cœur de nos questionnements durant les prochaines campagnes. À plus grande échelle, cette réflexion participe à un travail plus global dédié à l'évolution des stratégies de mobilité et à l'organisation des groupes humains au cours de la seconde moitié du Tardiglaciaire. Le matériel collecté en 2014 confirme l'idée d'une signature technique très spécifique. Cet assemblage est en effet particulièrement riche en pièces retouchées et ébréchées. Plus de $40 \%$ du matériel (hors tamisage) présentent des retouches. L'étude fonctionnelle n'ayant pas encore été réalisée, il est pour le moment difficile d'évaluer le taux de supports bruts ayant été utilisés sur le site. A contrario, les éléments pouvant témoigner d'activités de débitage (nucléus, pièces techniques, débris) restent particulièrement rares dans la série (un seul nucléus dont l'attribution à la composante azilienne n'est pas garantie). De même, l'analyse des pièces récoltées au tamisage montre un faible effectif de fragments de supports parmi le matériel de moins de $10 \mathrm{~mm}$. La plupart de ces petites pièces, souvent classées comme " esquilles », sont au Rocher de l'Impératrice des micro-éclats. Si une part de ces pièces a certainement été obtenue lors d'opérations de débitage, une partie de cet ensemble est clairement constituée d'éléments détachés lors de la retouche des supports et, notamment, lors de la fabrication des dos des armatures. Plutôt que de témoigner d'importantes activités de production de supports sur le site, l'analyse de ces micro-éclats semble plutôt suggérer la pré- sence d'activités de conception et d'entretien d'outils et notamment d'armatures.

La chaîne opératoire est donc particulièrement fragmentée sur ce site. C'est d'ailleurs également ce que suggère le protocole de remontage (un seul remontage de deux pièces dont un nucléus ne pouvant pas être rattaché avec certitude à la composante azilienne). Les activités de taille seraient donc présentes, mais en faible proportion au Rocher de l'Impératrice. La composition de l'assemblage suggère d'ailleurs des activités essentiellement tournées vers l'acquisition et le traitement de gibier. Les pointes de projectile dominent en effet largement avec plus de cent pièces (alors que le tamisage de 2014 n'a pas encore été traité), ce qui représente $42 \%$ de l'outillage et $10,5 \%$ de la totalité de l'assemblage (pièces relevées à la fouille et au tamisage de 2013). Ces pièces ont été, pour certaines, fabriquées sur place, comme en témoigne la présence d'ébauche et d'éclats de retouche de dos, ou abandonnées après la chasse, comme le montrent plusieurs éléments suggérant des impacts violents. Le reste de l'outillage est largement dominé par les lames à retouches rasantes ou semi-abrupte, dont une partie porte une troncature sur l'une de leurs extrémités. Même si cette hypothèse devra être vérifiée par l'analyse fonctionnelle, ces pièces pourraient avoir été utilisées pour des activités de découpe. À ces pièces s'ajoutent, en moindres proportions, des troncatures, des burins (essentiellement sur troncature) et de très rares grattoirs.

Plusieurs éléments suggèrent une occupation courte de l'abri par un groupe restreint. Les pièces présentant une altération thermique sont en effet rares $(8 \%)$ et, pour le moment, aucun élément ne permet de parler d'une structuration de l'espace ou de foyer. L'assemblage est de plus peu abondant. Les tranchées réalisées en 2014, afin de vérifier l'extension de l'occupation, suggèrent également une occupation limitée à une zone restreinte au fond de l'abri. Les groupes aziliens semblent être venus sur ce gisement, à une ou plusieurs reprises, déjà munis de tool kits constitués d'armes de chasse, d'outils divers et probablement de nucléus en cours de débitage, desquels auraient été extraits des supports à la demande, le nucléus étant potentiellement transporté vers un nouveau site.

Ce gisement, installé sur un point haut offrant un point de vue imprenable sur la paléovallée de l'Elorn et les autres fleuves comme l'Aulne, devait constituer un site de premier choix pour surveiller les circulations d'animaux dans cette grande vallée aujourd'hui submergée par la mer d'Iroise. Il semble avoir été utilisé (certainement à plusieurs reprises) par des groupes restreints, durant de courtes périodes, pour des activités essentiellement tournées vers l'acquisition et le traitement du gibier. Contrairement à Pont-Glas, d'autres activités ont certainement également été pratiquées, certes en moindre proportion, au Rocher de l'Impératrice. C'est ce que suggère notamment la présence des nombreuses plaquettes de schiste gravées. Il ne fait nul doute que l'analyse fonctionnelle de ce matériel apportera des précisions à ces conclusions préliminaires quant aux activités pratiquées sur le site. 


\section{VERS UNE TYPOLOGIE DES CAVITÉS EN DOMAINES PLUTONIQUES OU MÉTAMORPHIQUES}

\section{Nature des roches et processus érosifs}

Les fouilles évoquées dans le chapitre précédent n'ont concerné que des grottes et abris creusés dans des massifs granitiques ou des quartzites; il n'est question pour l'instant que de tirer des conclusions sur le potentiel de ces contextes archéologiques. Sous les effets de l'érosion, les roches plutoniques et métamorphiques n'ont engendré en Bretagne que des cavités de petites dimensions ou des surplombs peu profonds, quand bien même leurs plafonds sont très élevés. Seules les falaises marines font exception, car les coups de boutoirs de l'océan assurent un creusement et un déblaiement plus rapide que l'effondrement du plafond sous l'effet de la gravité et de l'érosion chimique. Lorsque ces cavités profondes sont déconnectées du milieu marin qui les a creusées, elles deviennent habitables par les groupes humains, qui viennent y chercher un toit, des parois ou des matériaux à tailler lorsque des galets jonchent le sol. C'est le cas de Ménez Drégan au début de plusieurs régressions marines, notamment le stade 12 pour la couche 9 (MD9) ou le stade 10 pour les couches 4 et 5 (MD4 et MD5). C'est donc pour l'essentiel lors des périodes glaciaires qu'elles furent occupées et plutôt dans leur partie initiale, avant que les dépôts de pente ne viennent en assurer le colmatage. Il y a donc deux domaines géographiques bien distincts pour les cavernes armoricaines en fonction des processus érosifs qui y dominent.

La nature des roches influe évidemment aussi sur la configuration de ces cavités. Pour les grès-quartzites anciens, d'âge primaire, les cavités du domaine continental correspondent quelques fois à des failles profondes comme la grotte de Roc'h Toul, mais le plus souvent à des pieds de falaise à faible surplomb comme le Rocher de l'Impératrice. Ces roches se fragmentent en gros blocs anguleux, formant parfois dans certains secteurs des éboulis secs avec peu de matrice fine.

Dans les massifs de granite, les abris-sous-roche en pied de falaise sont également de faible profondeur, ainsi le Goaréva où le surplomb atteint certes les neuf mètres de haut, mais où il ne couvre le sol que sur deux à trois mètres (fig. 13). Sa formation géologique est originale, puisque la zone abritée correspond à un filon de dolérite érodé entre deux zones de granite; les habitats du Paléolithique moyen s'y sont implantés sur une surface d'environ $80 \mathrm{~m}^{2}$. Mais ce sont les différentes formes d'érosion de cette roche qui fournissent le plus large éventail de cavités pour l'instant recensées, toujours de petites dimensions (fig. 14). Le granite est une roche magmatique grenue, certes résistante, mais peu souple et donc très faillée. L'eau qui parcourt les réseaux de diaclase agit à la fois par l'hydrolyse de certains minéraux (micas noirs, feldspaths) et par l'hydratation (sans modification chimique) qui désolidarise les minéraux. Les minéraux de quartz résiduels composent l'essentiel de l'arène gra- nitique, un sable grossier qui contient aussi certains feldspaths et des argiles. Lorsque ces éléments sont emportés par l'eau, subsistent des boules rocheuses qui dépassent souvent la dizaine de mètres, qui forment des chaos sur les pentes. La configuration en « châteaux forts » au sommet des coteaux est moins fréquente dans le Massif armoricain, peut-être parce que leur accessibilité en a fait des cibles de choix pour l'implantation de carrières de pierre. Plusieurs configurations sont adéquates pour les occupations humaines, comme nous pouvons le constater dans le massif de Huelgoat (Finistère) ou dans celui de Quintin (Côtes-d'Armor) : cavité creusée par la météorisation (qualifiée de Taffoni par les géomorphologues), rebord basculé d'un rocher enraciné dans l'affleurement en place (qualifié de Tor), surplomb d'une grande boule sur une plus petite ou encore basculement d'une boule supérieure sur une autre. Cette dernière configuration très fréquente en Bretagne est notamment celle de Pont-Glas.

À cette brève typologie, il faudrait ajouter - au moins comme sites potentiels - les couloirs de fragmentation des boules granitiques, larges parfois de quelques mètres et qui offrent des parois naturelles droites aisées à aménager (tabl. 1). Cette grande diversité des abris offerts par les massifs granitique est hélas altérée par les immenses destructions menées par les carriers depuis des millénaires, avec une intensification particulière durant les deux derniers siècles. Avec la mécanisation des pratiques agricoles, il est aussi commun de trouver des empilements artificiels de blocs au milieu d'une parcelle, un alignement de boules dans un talus ou encore la mention orale de chaos granitique totalement arasés ou enterrés aujourd'hui, si bien qu'il est presque impossible de reconstituer le maillage initial du paysage. Si l'abri de Pont-Glas né du basculement de deux boules granitiques est parvenu jusqu'à nous, c'est probablement dû à son « fil » inapproprié nous a confié un ancien tailleur de pierre; le bloc de plus de $20 \mathrm{~m}$ de diamètre dans lequel vient se nicher l'abri de Kerbizien a été scié au début des années 1980 et n'a été préservé que par l'intervention énergique de son propriétaire. Quant aux chaos rocheux de la "rivière d'Argent» à Huelgoat, on ne sait pas au juste si c'est leur difficulté d'accès pour une exploitation industrielle ou leur aspect pittoresque qui leur a permis de subsister jusqu'à aujourd'hui, pour devenir une attraction touristique majeure de cette région.

Les schistes si abondants dans le Massif armoricain livrent également de petits surplombs dans les falaises et de grandes grottes en contexte littoral. Le meilleur exemple archéologique provient de la presqu'île du Cotentin, à la pointe du Rozel dans la Manche (Van Viet et al., 2006). Un abri au pied d'une falaise marine en schiste paléozoïque a été occupé en début du Dernier Glaciaire (vers $115 \mathrm{ka}$ ), alors qu'un épisode de refroidissement climatique et de régression marine en assurait l'accès. L'ensevelissement ultérieur de cette falaise par un massif de sables dunaires recouvert d'un head protecteur a assuré un colmatage exceptionnel de cette cavité. Deux occupations successives du Paléolithique moyen y ont été fouillées sous la direction de F. Scuvée dans les années 1960. 


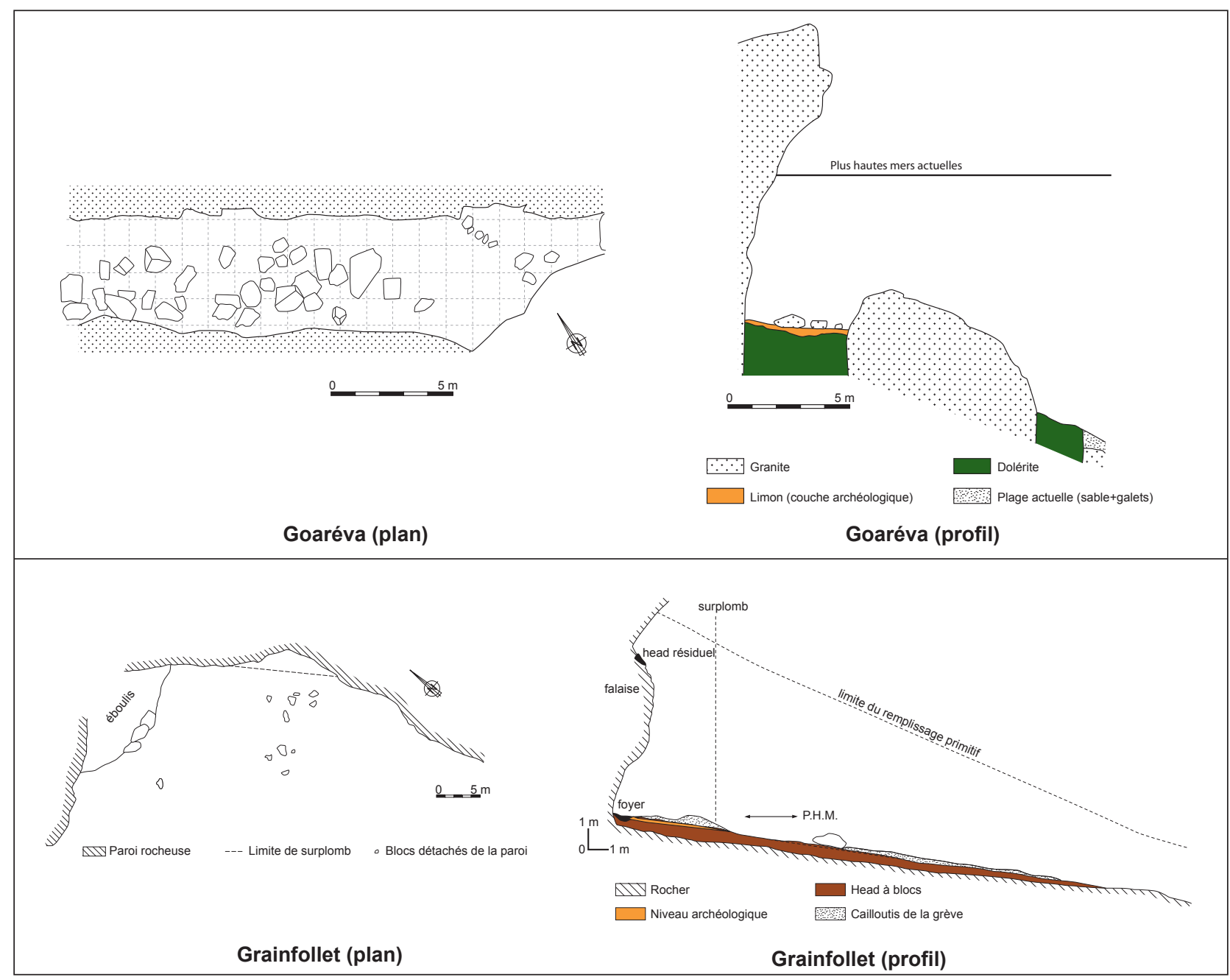

Fig. 13 - Plans et profils des abris-sous-roche paléolithiques de Grainfollet, Saint-Suliac, Ille-et-Vilaine, et de Goaréva, Bréhat, Côtesd'Armor (DAO L. Quesnel et G. Marchand, d'après Monnier, 1980).

Fig. 13 - Plans and profiles of the Palaeolithic rock-shelter of Grainfollet, Saint-Suliac, Ille-et-Vilaine, and Goaréva, Bréhat, Côtesd'Armor (CAD L. Quesnel and G. Marchand, after J.-L. Monnier, 1980).

Les vestiges archéologiques consistaient d'une part en une industrie lithique (éclats Levallois et éclats allongés dans le niveau inférieur, éclats et lames dans un niveau supérieur), d'autre part en des restes d'une grande faune de mammifères. Hormis ce site de Basse-Normandie fossilisé par des dunes, les abris-sous-roche et grottes dans les domaines schisteux restent à mieux documenter et ils représentent un potentiel important à l'échelle du Massif armoricain.

Les formations calcaires de l'ère primaire (Carbonifère et Dévonien) sont très rares sur le Massif armoricain, mais elles ont comme ailleurs engendré des formations karstiques propices aux occupations humaines. Les plus notables sont à l'est du Massif en Mayenne, dans la vallée de l'Erve (canyon de Saulges), dans des réseaux creusés aux dépens du Calcaire de Sablé (Tournaisien supérieur à Viséen moyen). Parmi les nombreuses grottes, les grottes Rochefort et de la Chèvre recèlent une succession d'occupations paléolithiques, tandis que les grottes Margot et de Mayenne-Sciences sont justement célèbres pour leurs figurations pariétales (Gravettien et Magdalénien supérieur). À ce jour, ce sont les seules cavités ornées du Massif armoricain. À l'extrême ouest, les formations calcaires de Plougastel-Daoulas (Gédinnien supérieur Siegénien moyen) sont également parcourues de réseaux profonds, sans occupations préhistoriques attestées pour le moment; il est vrai qu'elles ont été fortement dégradées par des carrières et des aménagements plus récents...

\section{Nature et potentiel des remplissages sédimentaires}

L'alimentation des dépôts sédimentaires protégés par les couvertures rocheuses se fait comme de juste par les produits mêlés de la désagrégation des parois et des apports extérieurs, mais les équilibres semblent différents suivant les conditions climatiques et l'orientation de la cavité. Durant l'Holocène, les processus thermoclastiques si vigoureux par exemple en domaine montagnard sont très faibles à l'évidence en France atlantique, ce qui explique à la fois les creusements assez peu prononcés des blocs 


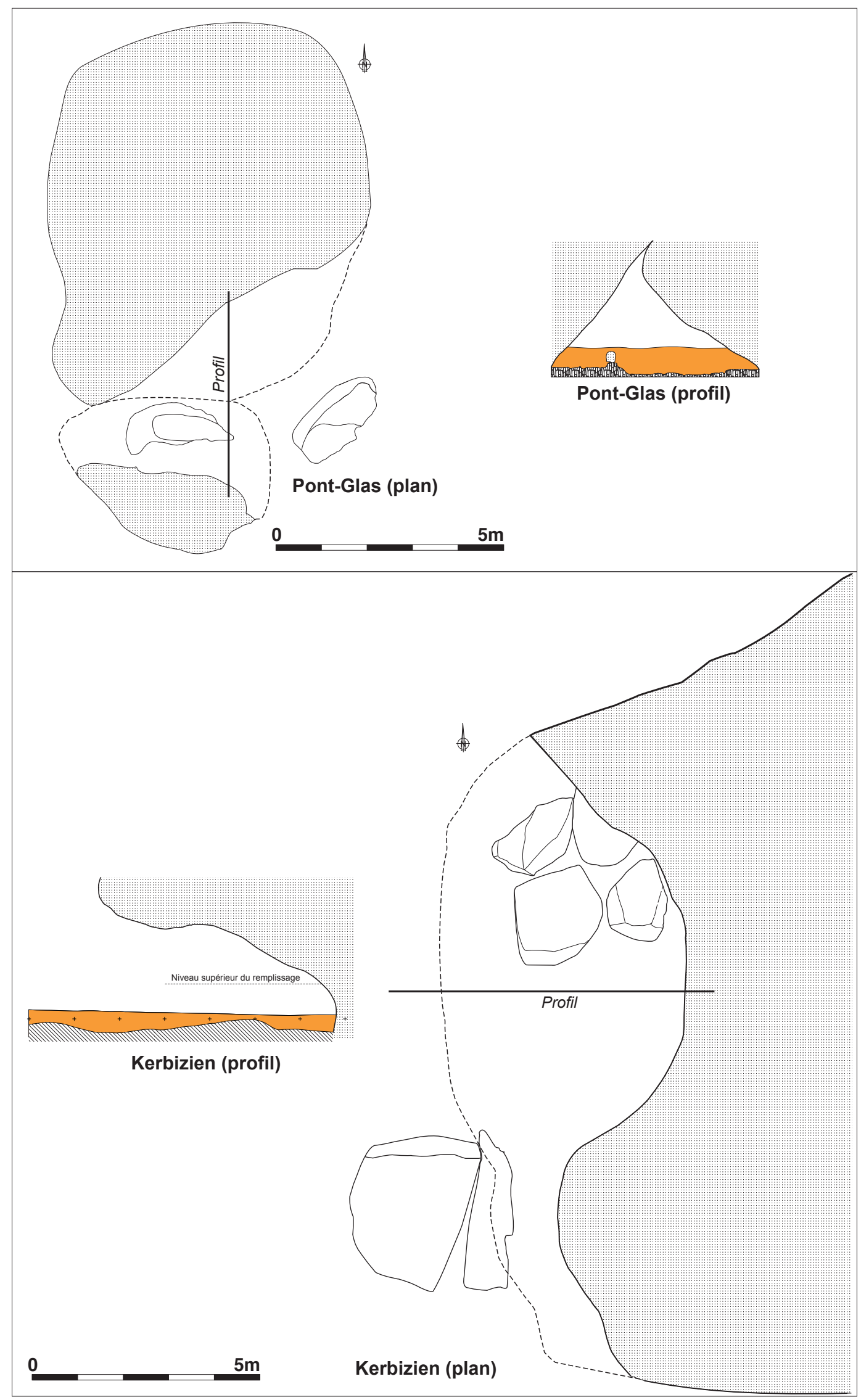

Fig. 14 - Plans et profils des sites de Pont-Glas, Plounéour-Ménez, et Kerbizien, Huelgoat (DAO G. Marchand).

Fig. 14 - Plans and profiles of the rock-shelter of Pont-Glas, Plounéour-Ménez, and Kerbizien, Huelgoat (CAD G. Marchand). 


\begin{tabular}{|l|l|l|}
\multirow{2}{*}{$\begin{array}{l}\text { Type } \\
\text { Pied de falaise }\end{array}$} & Sous-type & Exemple archéologique \\
\hline \multirow{3}{*}{ Faille } & Abri de pied de falaise & Goaréva, Grainfollet, le Rocher de l'Impératrice, le Rozel \\
\hline \multirow{2}{*}{ Karst } & Grotte de faille & Roc'h Toul \\
\cline { 2 - 3 } & Couloir d'érosion marine & Ménez Drégan \\
\hline \multirow{3}{*}{ Blocs } & Grotte & Grottes de la vallée de l'Erve \\
\hline & Couloir de fragmentation & - \\
\cline { 2 - 3 } & Boule en surplomb & - \\
\cline { 2 - 3 } & Boule basculée & Pont-Glas \\
\cline { 2 - 3 } & Taffoni (météorisation) & Kerbizien \\
\hline
\end{tabular}

Tabl. 1 - Typologie des cavités rocheuses du Massif armoricain.

Table 1 - Typology of the Rock-Shelter on the Armorican Massif.

et les remplissages constitués principalement d'éléments fins. Dans les abris en grès-quartzite comme le Rocher de l'Impératrice, les éboulis massifs semblent pléniglaciaires, mais un nappage lœssique est également observé. Dans les abris granitiques, l'essentiel de l'apport provient des parois à Pont-Glas, tandis que des passées limoneuses s'intercalant avec l'arène granitique sont notées dans le niveau tardiglaciaire résiduel de Kerbizien. En Bretagne du Nord durant le Pléniglaciaire, le remplissage par des lœss vient compenser cet apport sédimentaire endogène assez faible, notamment pour les abris s'ouvrant vers le nord, mais aussi autour des rochers du littoral (Monnier, 1980). Les vents ont même porté ces poussières au-delà des crêtes des monts d'Arrée, où elles ont été s'accumuler dans l'abri de Kerbizien et au sud de cet énorme bloc (fig. 8). Dans de nombreux abris en chaos granitique que nous avons simplement visités ou dans ceux déjà sondés en 2008 à quelques dizaines de mètres de Pont-Glas (chaos de Tri-dorchenn, Dorchennic et Viou Euzc'hlazard, sur la même commune), l'apport de colluvions récentes est remarqué, mais elles signalent souvent un lessivage des niveaux : c'est une des limites évidentes de ces chaos granitiques, nés du ruissellement des eaux.

Des phases d'érosion avec vidange totale peuvent être notée à la base des stratigraphies dans les deux abris granitiques intégralement fouillés présentés ici : à Kerbizien cela se passerait avant le dépôt des lœss (donc avant le Pléniglaciaire), à Pont-Glas au moins avant le Mésolithique. L'accumulation de l'arène granitique atteint ensuite moins d'un mètre à Kerbizien, un demi-mètre à Pont-Glas, ce qui reste bien entendu faible. Quelle est leur valeur en termes d'enregistrement archéologique? Ces stratigraphies sont fragiles car elles sont constituées de matériaux meubles, mais aussi parce que ces espaces ont pu être fréquemment réoccupés. L'activité des animaux fouisseurs, notamment au fond des cavités, n'est pas anodine. Elle additionne ses effets aux ruissèlements le long des blocs, qui créent ici comme dans les cavités karstiques des " effets de parois ». Dans ces zones de mélanges verticaux larges de 10 à $20 \mathrm{~cm}$, s'agglomèrent toutes les composantes culturelles reconnues ailleurs en stratigraphie. À l'opposé de l'abri, à proximité de la source de lumière, les racines et radicelles sont nom- breuses et participent au démembrement de la roche de manière active, l'activité organique brouillant d'ailleurs les limites entre les différentes couches. Ces phénomènes classiques dans l'archéologie des grottes atteignent des seuils dramatiques dans les plus petites de ces cavités.

Ces limites sédimentaires doivent évidemment être bien intégrées à l'heure d'analyser la composition des assemblages lithiques que l'on y recueille. La possibilité parfois de collecter la totalité des objets abandonnés dans ces petites cavités - c'est le cas à Pont-Glas - vient contrebalancer quelque peu ces problèmes sédimentaires.

\section{LES CAVERNES ARMORICAINES DANS LEURS RÉSEAUX ÉCONOMIQUES}

\section{Les outils d'analyse à notre disposition pour percevoir des caractères fonctionnels particuliers}

À l'exception notable de la grotte marine de Ménez Drégan, la modicité des remplissages sédimentaires des cavernes armoricaines ne donne pas prise à des modélisations chronologiques de grande ampleur pour juger de la succession des industries humaines. L'intérêt qu'on leur porte est davantage dans la complémentarité fonctionnelle que leurs contenus archéologiques nous offrent en regard des sites de plein air. La liste des éléments de distinction doit être établie, avant d'évoquer leur potentiel informatif pour la constitution de modèles de fonctionnement.

En ce qui concerne les structures d'habitat, aucun élément évident ne semble ressortir des premières explorations, sinon évidemment... l'absence de structures lourdes et d'aménagement de l'espace! Cette observation doit nécessairement être comparée aux pratiques contemporaines des occupations et modulée par les faibles surfaces encore explorées. La densité des vestiges apparaît comme un paramètre d'usage difficile, qui dépend de la durée d'occupation, une donnée rarement disponible avec suffisamment de finesse.

En ce qui concerne la nature des vestiges recueilli, la vingtaine d'éléments de plaquettes gravées du Rocher de 
l’Impératrice est révélatrice d'un comportement encore unique sur le Massif armoricain pour le Tardiglaciaire. Peut-être faut-il considérer comme un faible écho de ces pratiques symboliques les 166 fragments de schiste exhumés lors de la fouille de Kerbizien, même si ils ne portaient aucune trace anthropique? La place de telles activités dans les modes de vie au Paléolithique final doit être interrogée, ce qui renvoie à une gamme de questions somme toute classiques pour le Paléolithique supérieur.

Les différentes proportions de restes lithiques reflètent directement la segmentation des chaînes opératoires dans l'espace ou pour dire autrement la dimension spatiale des processus de taille et de fabrication des outils. C'est dans ce registre que nous avons enregistré les résultats les plus pertinents pour notre problématique fonctionnelle. En ce qui concerne l'acquisition des matériaux, ce n'est pas tant l'origine des matériaux que leurs modes d'introduction dans le gisement qui nous semblent originaux, toujours en comparaison avec les pratiques en usage à la période considérée. À Kerbizien et au Rocher de l'Impératrice, dans l'Azilien ancien, le clair déficit en produits corticaux révèlerait une introduction des supports ou des nucléus déjà préformés. Dans le premier et le second Mésolithique de Pont-Glas, les éclats corticaux et semi-corticaux représentent $23 \%$ des éclats entiers contre 56\% à Beg-er-Vil (Quiberon, Morbihan) ou $44 \%$ à Beg-an-Dorchenn (Plomeur, Finistère), deux vastes sites à niveaux coquilliers considérés comme des habitats pérennes (Marchand, 2014). Cette rareté des produits corticaux se conjugue à une grande diversité non seulement des roches, mais aussi de leurs faciès, qui traduirait une introduction dans l'habitat de manière très fractionnée dans le temps (Marchand et al., 2011). La rareté des nucléus sur ce site, voire leur absence sur les deux sites aziliens, laisserait penser à une poursuite du débitage hors de l'abri. C'est donc par la segmentation importante des activités dans l'espace que ces assemblages issus des cavités armoricaines se distinguent en premier lieu, avec une surreprésentation des séquences de plein débitage, mais aussi de confection des outils et de réaffûtage.

Le type d'outils recueillis dépend évidemment de manière directe des activités réalisées, mais en l'absence d'études tracéologiques les supports aménagés n'en sont qu'un faible reflet pour le Paléolithique final et le Mésolithique, où les outils a posteriori dominent d'ordinaire. Le degré de destruction des armatures de chasse est une donnée intéressante à retenir, tant les traces d'impact sur les pièces abandonnées que les pièces cassées à l'impact : il est particulièrement évident à Pont-Glas. L'entretien fréquent des burins à l'Azilien est aussi indicateur fonctionnel intéressant, d'autant plus lorsque ces outils manquent à l'appel comme à Kerbizien. Dans ce cas, l'hypothèse d'une importante mobilité des individus peut être émise. L'abandon de grandes lames - dans un contexte généralisé de pénurie des matériaux sur le Massif armoricain est un élément à retenir, qui reflète à la fois la conception que les humains se sont faits d'un outil (et d'une fonction) et une mobilité importante qui ne s'embarrasse pas d'un objet non fonctionnel. L'absence de grattoirs, à Kerbizien, et leur rareté au Rocher de l'Impératrice, tra- duiraient une faible importance des activités auxquelles ils sont liés (traitement des peaux?) car d'ordinaire ces outils sont nombreux au début de l'Azilien.

Enfin, l'altération des pièces lithiques par les chocs thermiques peut être aussi convoquée comme élément discriminant entre les sites. Un temps d'occupation prolongé devrait engendrer des taux plus importants. Cependant, au même titre que les autres paramètres, il est d'interprétation ambigüe et doit être modulé par ce que l'on connait des autres contextes archéologiques. Dans les trois fouilles les plus récentes mentionnées ici, le taux de pièces brûlées est nettement inférieur à celui des gisements contemporains : sur le silex exclusivement, il est de 7,5\% à Kerbizien, $8 \%$ au Rocher de l'Impératrice et 26,5\% à Pont-Glas.

Il semble pour l'instant que les éléments de différenciation fonctionnelle soient nettement plus ténus entre les sites du Paléolithique supérieur armoricain, faute probablement de travaux récents en ce sens. Pour le Néolithique également, de telles perspectives font encore défaut. Malgré les importants bouleversements stratigraphiques qui y furent reconnus, l'abri-sous-roche de la Jupinerie, à Omonville-la-Petite en Basse-Normandie, fouillé par C. Marcigny et L. Juhel, a livré de nombreuses armatures du Néolithique ancien, qui orientent l'une des interprétations vers l'hypothèse d'une halte de chasse (Juhel et al., 2006; Juhel et Marcigny, 2008; Charraud, 2012; Ghesquière, 2012). Mais cet abri en contexte plutonique (Monzogranites et granodiorites d'Auderville et de Thiébot) reste bien isolé en regard des centaines d'habitats ou de sites funéraires référencés dans le Néolithique de l'Ouest à ce jour et tout reste à écrire sur d'éventuelles économies de chasse dans le Néolithique atlantique. À l'heure d'insérer les cavités armoricaines dans les modèles de peuplement préhistoriques, nous ne proposerons donc ici que deux cas de figures, l'un pour les sociétés du Tardiglaciaire, l'autre pour celles du début de l'Holocène.

\section{Petites cavités et modèles de peuplement au Tardiglaciaire}

L'inventaire des cavernes présenté dans cet article montre un clair hiatus dans les occupations sous roche entre la fin du Paléolithique moyen et le milieu du Tardiglaciaire. De manière générale d'ailleurs, les sites attribués au Paléolithique supérieur ancien sont rares sur le Massif armoricain et aucun indice d'occupation n'est aujourd'hui disponible entre le Solutréen et l'Azilien ancien. La plupart des gisements autrefois attribués au Magdalénien ont effet été rajeunis ces dernières années pour être basculés à la transition Pléistocène-Holocène (Marchand et al., 2004 ; Naudinot, 2010 et 2013a).

Deux hypothèses peuvent-être proposées pour expliquer ce phénomène : soit une destruction des sites par divers phénomènes érosifs, soit une désertion du Massif armoricain au cours du Pléniglaciaire et des premiers temps du Tardiglaciaire, suivi d'un repeuplement progressif à partir de la fin du Bølling. La première hypothèse doit être testée en établissant une cartographie des processus sédimentaires à l'échelle régionale au cours de 
cette période. En parallèle, la mise en place d'un système d'information géographique (SIG) visant à analyser les positions topographiques des sites et leur contexte géomorphologique est en cours de construction. En attendant ces développements, la seconde hypothèse ne semble pas inenvisageable. L'Ouest est en effet certainement une des parties de la France où la dynamique de recherche par les archéologues non professionnels est la plus développée. Or, aucune des milliers de séries découvertes lors de ces prospections ne livre le moindre indice pouvant être rapporté au Magdalénien. Dans l'hypothèse où ces sites auraient été balayés par des phénomènes érosifs, il y a fort à parier que quelques pièces auraient été découvertes en position secondaire dans certains secteurs.

Le Massif armoricain pourrait ainsi avoir été déserté par les groupes paléolithiques au cours du Pléniglaciaire et des premiers temps du Tardiglaciaire. La grotte de Rochefort en Mayenne, sur les marges orientales du Massif armoricain, seul site montrant une succession d'occupations du Paléolithique supérieur dans la région, montre d'ailleurs un hiatus entre le Solutréen et la transition Dryas récent-Préboréal. On notera toutefois la présence d'une bipointe certainement attribuable à l'Azilien ancien dans les niveaux remaniés du site et la présence de témoignages artistiques attribués au Magdalénien supérieur (Hinguant et al., 2012). Des occupations dans les zones aujourd'hui submergées sont en revanche possibles, d'autant plus que certains secteurs, comme la paléovallée du fleuve Manche devaient constituer des axes de circulation privilégiés pour le gibier et les hommes. Cette grande plaine regorgeait également de ressources minérales, sous la forme d'affleurements de silex, dont l'absence sur le Massif armoricain a pu participer à écarter cette région des circuits économiques magdaléniens. L'existence de groupes pratiquant des économies de prédation marine est également très probable. Les recherches sur les causes de cette désertion du Massif armoricain par les groupes magdaléniens et sur les modalités de repeuplement de ce territoire n'en sont qu'à leurs balbutiements et elles constituent un de nos axes de recherche privilégiés.

Une nouvelle dynamique de peuplement semble progressivement se mettre en place au cours du Tardiglaciaire, avec l'occupation de quelques sites par des groupes de l'Azilien ancien. Ils sont pour la plupart localisés dans des grottes et des abris-sous-roche (Roc'h Toul, Kerbizien et le Rocher de l'Impératrice pour les plus significatifs), même si quelques rares bipointes ont été découvertes au gré des ramassages de surface dans le Nord du Finistère. Pourquoi cette nette prédominance des abris au cours de l'Azilien ancien, alors que les très nombreux habitats attribuables à la phase récente de ce techno-complexe sont en majorité des gisements de plein air en zones basses (Naudinot, 2010)? Deux scénarios peuvent être pour le moment envisagés pour répondre à cette question.

Le premier modèle, désigné ici comme « modèle pionnier », s'appuie sur les résultats des fouilles des gisements de Kerbizien et du Rocher de l'Impératrice, qui présentent des signatures techniques particulières suggérant des activités orientées. Ces sites pourraient ainsi illustrer des incursions de petits groupes à forte mobilité résidentielle sur le Massif armoricain, depuis la plaine de la Manche ou les bassins sédimentaires voisins, peut-être pour des activités particulières. Le véritable repeuplement du Massif armoricain n'interviendrait que plus tard, lors de la phase récente de l'Azilien, pour laquelle les informations sont beaucoup plus nombreuses et dont les indices à disposition semblent suggérer un système économique plus complet. Un des indices souvent mis en avant pour distinguer ces peuplements dits pionniers est le transport par les groupes d'une proportion importante de matériaux allochtones sous la forme d'un équipement polyvalent et évolutif (Kelly et Todd, 1988; Tolan-Smith, 2003). Compte tenu du faible nombre de sites encore disponibles dans la région, il est difficile de chercher à tester ce modèle. Notre réflexion est d'ailleurs largement limitée par notre connaissance encore trop lacunaire des silex des gisements de la Manche et par notre incapacité, en l'absence de cortex, à différencier une pièce débitée dans un rognon de silex directement extrait des gisements crétacés aujourd'hui submergés, d'un support produit à partir d'un galet arraché à ces affleurements et drainé par l'océan sur les nombreux cordons littoraux. Il est ainsi encore impossible de déterminer avec certitude si les lames découvertes à Kerbizien ou au Rocher de l'Impératrice ont été produites à partir de matériaux sub-locaux ou sur des silex allochtones. Plusieurs indices, dont les dimensions de certains supports ou la présence de plages corticales non altérées sur quelques pièces, semblent toutefois suggérer un usage important de volumes sans équivalent dans les cordons littoraux et dont l'origine pourrait être les affleurements de la Manche ou des cordons aujourd'hui submergés et localisés à proximité des gisements crétacés. De rares pièces en silex allochtones ayant circulé sur plus de $300 \mathrm{~km}$ ont en revanche été identifiées sur les deux sites, ainsi qu'à Roc'h Toul. La nature du matériel retrouvé dans ces deux abris finistériens suggère l'apport d'un équipement personnel. Les témoins d'activités de débitage sont ainsi rares et tout porte à croire que les occupants de l'Azilien sont arrivés dans ces abris munis de leur outillage et de quelques nucléus en cours de réduction, qu'ils ont emporté ailleurs. Une part des outils semblent également avoir été déplacés hors du site (ou hors des zones fouillées?), comme l'illustre la disproportion entre le nombre de chutes et de burins à Kerbizien par exemple.

Le modèle " d'implantation pérenne » quant à lui considère les abris finistériens comme des sites aux activités orientées, intégrés dans un système de mobilité à dominante logistique. En considérant ce modèle, les sites d'habitat résidentiels seraient pour le moment inconnus. Ils pourraient avoir été installés dans des fonds de vallées et seraient aujourd'hui recouverts par une sédimentation trop importante pour autoriser leur visibilité.

\section{Petites cavités et modèles de peuplement au Mésolithique}

Les deux principales phases du Mésolithique - premier et second Mésolithique - ont été identifiées dans les cavités armoricaines, à Kerbizien dans les déblais et de meilleure manière à Pont-Glas. Elles correspondent à deux modèles 
bien distincts d'organisations socioéconomiques, en Bretagne du moins (Marchand, 2014).

Les informations concernant le premier Mésolithique commencent vers 8200 avant notre ère et couvrent le début du VII ${ }^{\mathrm{e}}$ millénaire. Il est frappant de constater l'absence d'observation de structures d'habitats en matériaux minéraux pour cette période en Bretagne même lors des grands décapages de l'archéologie préventive (Blanchet et al., 2002; Nicolas et al., 2012). Il en allait de même pour les sondages manuels, nécessairement plus limités en surface, qui ont été réalisés depuis les années 1970 (Berrou et Gouletquer, 1973; Yven, 2003a; Marchand, 2005). Les bases de données actuelles enregistrent donc des centaines d'habitats mésolithiques de plein air, notamment à l'extrémité de la péninsule, qui sont matérialisés par des pièces lithiques étalées sur de vastes superficies et dont on ne saurait dire bien évidemment s'ils procèdent d'occupations de grands ou de petits groupes. L'absence de structures lourdes en Bretagne conduit peut-être de manière fallacieuse à y voir un indice de haute mobilité (Marchand, 2014), alors que l'archéologie préventive ou programmée ne sait pas encore révéler la nature réelle des habitats mésolithiques. En Normandie en revanche, l'usage répété de sites de sommets de falaise se matérialise par des zones de combustion davantage aménagées (Ghesquière et al., 2000; Ghesquière, 2012). Les sites à grands foyers empierrés, tel l'Onglais à Acquigny dans l'Eure, sont également connus en domaine continental (Chancerel et Paulet-Loccard, 1998; Souffi, 2004). Et sur le Massif armoricain, la fouille de l'abri de la Jupinerie à Omonville-la-Petite (Basse-Normandie) a livré huit armatures du premier Mésolithique, en contexte fort remanié (Ghesquière, 2012, p. 198). Une telle diversité apparaît également en Poitou-Charentes, avec dans le vallon de la Pierre-Saint-Louis (Geay, Charente-Maritime) de nombreux foyers empierrés disposés dans des cuvettes et réalisés sur une longue période (Foucher et al., 2000), des occupations logistiques en abri-sous-roche à Bellefonds (Patte, 1971; Michel, 2011) ou une nécropole à inhumations à la fois collectives et multiples à la Vergne (Duday et al., 1998). Il n'y a pas de raison de penser toutefois que tous les modèles économiques du premier Mésolithique sont identiques dans ces régions et sur une aussi longue période. En Bretagne, les données archéologiques disponibles permettent juste de différencier ces vastes sites de plein air et les abris-sous-roche. Pont-Glas a une signature de site logistique, tandis que les strates détruites de Kerbizien, particulièrement riches en vestiges d'après les tas de déblais, laissent penser à de plus amples occupations.

La situation change nettement au second Mésolithique, tout du moins en Bretagne où les données sont les plus nombreuses. C'est en effet la période où les registres archéologiques littoraux apparaissent dans les corpus d'habitats mésolithiques. Avec la stabilisation du niveau des océans à une quinzaine de mètres sous l'actuel à la fin du VII ${ }^{\mathrm{e}}$ millénaire, on dispose enfin d'une fenêtre d'observation sur la bande littorale de l'époque, les fameux niveaux coquilliers du Sud de la région livrant des informations incontournables sur l'organisation des économies de prédation ou les fonctions des habitats. Le début du VII ${ }^{\mathrm{e}}$ millénaire est mal défini, avec les sites essentiels mais très dégradés par l'érosion de la pointeSaint-Gildas (Préfailles, Loire-Atlantique). En revanche à partir de 6200 avant notre ère et jusqu'à 5300, les fouilles des niveaux coquilliers de Beg-er-Vil (Quiberon, Morbihan), Téviec (Saint-Pierre-Quiberon, Morbihan), Hoëdic (Morbihan) et Beg-an-Dorchenn (Plomeur, Finistère) ont établi l'existence d'un très grand nombre de structures, qu'elles soient domestiques (aires empierrées, foyers empierrés, foyers en fosse, foyers à plat) ou funéraires (fosses et massifs de pierre). Les travaux sur les restes osseux révèlent par ailleurs le large éventail des proies et l'occupation possible en toutes saisons sur ces sites littoraux. Enfin, l'analyse géochimique des ossements humains découverts dans les cimetières de Téviec et Hoëdic a permis d'assoir l'hypothèse de populations très inféodées aux ressources maritimes (Schulting et Richards, 2001). À proximité de ces grands habitats, il existe également de petites stations de sommets de falaise moins bien décrites, qui se signalent par des pièces lithiques sur quelques mètres carrés.

Les sondages réalisés sur les nombreux sites de plein air à l'intérieur de la péninsule n'ont en revanche livré aucune structure empierrée (Marchand, 2005 et 2009), mais beaucoup d'informations sur les chaines opératoires de débitage et sur les modes d'acquisition des matériaux. Ces sites occupent toujours de très larges superficies et livrent des centaines de milliers d'objets. L'hypothèse d'une dichotomie entre la bande littorale et la zone continentale a progressivement germé (Marchand, 2007 et 2014). Dans ce modèle dual, ses occupations discrètes feraient de Pont-Glas le parangon de la station logistique continentale, au milieu des collines du centre de la péninsule, avec une signature technique originale : très forte diversité des roches et de leurs faciès (représentés par quelques éléments à chaque fois), rareté des phases initiales du débitage, quasi-absence des nucleus, surreprésentation des séquences lamellaires dans le débitage, large domination des armatures dans l'outillage aménagé et dommages très importants sur ces armatures (Marchand et al., 2011).

Quels que soient les modèles économiques adoptés, au début comme à la fin du Mésolithique, l'abri-sousroche de Pont-Glas s'annonce comme un type très particulier, qu'il conviendrait d'étoffer pour améliorer ou au contraire atténuer sa valeur paradigmatique. Les données fonctionnelles au sens large à notre disposition pour la fin du Mésolithique en Bretagne sont aujourd'hui assemblées dans un modèle logistique, qui se développe différemment dans les zones continentales et littorales.

\section{UNE NOUVELLE DYNAMIQUE DE RECHERCHE DANS LE NORD-OUEST DE LA FRANCE}

es fouilles réalisées récemment sur trois abris-sousroche à l'ouest du Massif armoricain sont venues 
bousculer nos connaissances sur les réseaux économiques de la fin du Paléolithique et du Mésolithique, en livrant des industries lithiques à la structure fonctionnelle très originale. Comme nous l'attendions, une signature d'habitat logistique est apparue dans des cavités de petite superficie, qui ne pouvaient pas abriter de toutes les manières plus d'une poignée d'êtres humains. Nos outils d'analyses de la segmentation des activités dans l'espace sont encore grossiers; l'usage de chacun de ces indicateurs (proportions de produits corticaux ou d'outils, taux de pièces affectées par les chocs thermiques, ...) n'a de sens qu'en valeur relative à une époque donnée. C'est leur association qui est d'ailleurs pertinente. La compréhension des occupations dans les petites cavités nécessite en tous les cas de mettre à plat la question de l'interprétation par l'archéologue de la mobilité collective au cours de la Préhistoire; c'est un immense chantier qui va évidemment bien au-delà des travaux présentés ici.

Il conviendra aussi d'affiner ces observations par d'incontournables travaux de terrains, car des différences ne manqueront pas d'apparaître entre ces modes d'occupation suivant la chronologie. La question de l'absence pour l'instant totale de sites du Paléolithique supérieur dans le centre de la Bretagne pourra-t-elle alors être réglée? Les travaux remarquables menés dans les grottes de la vallée de l'Erve en Mayenne viennent conforter l'hypothèse d'une occupation de ces contrées atlantiques durant certaines phases du Paléolithique supérieur, même parmi les plus rigoureuses comme au Solutréen (Biard et Hinguant, 2015). Il en va de même sur le littoral nord de la Bretagne, même si les datations des assemblages du Paléolithique supérieur mériteraient assurément d'être précisées. L'usage de ces cavités lors du Néolithique reste aussi une question à traiter. L'abri-sous-roche de la Jupinerie a montré un visage original du Néolithique ancien en abri-sous-roche, mais on peut aussi envisager l'existence de pratiques funéraires en grotte, comme en HauteNormandie (Poulain, 1928) ou en Poitou (Patte, 1971). Il est à craindre dans ce cas que la dissolution très fréquente des ossements n'entrave cette identification.

À terme, un inventaire de ces cavités en domaine plutonique ou métamorphique reste à réaliser sur le Massif armoricain en lien avec les services archéologiques nationaux et départementaux, à des fins de préservation et d'exploitation scientifique raisonnée. Au même titre que les chambres mégalithiques, ce sont des sites fragiles, que quelques coups de pioche peuvent définitivement oblitérer. Un tel programme gagnera à être organisé par types de massif géologique et par mode d'érosion. On a vu que le littoral est à considérer à part, car l'érosion mécanique l'emporte largement en vitesse et en puissance sur l'altération chimique liée aux infiltrations d'eau. Et dans ce cas, seules les anciennes cavités perchées dans les falaises et déconnectées du battement des marées offrent un potentiel intéressant. Les recherches doivent aussi s'attacher aux substrats schisteux à la vitesse de remplissage peut-être assez élevées et pourtant très mal connus du point de vue de l'archéologie dans cette partie de la France. En trouvant abri dans les cavernes, l'archéologue armoricain doit prendre le temps, lui aussi, de confectionner ses outils.

Remerciements : Nous remercions Michel Le Goffic pour sa collaboration précieuse lors de ces différents travaux, Dominique Sellier pour ses réflexions concernant les formes d'érosion granitiques, Klet Donnart et Tony Bulger pour leur créativité, ainsi que deux rapporteurs anonymes pour leurs corrections et les nouvelles informations qu'ils nous ont apportées.

\section{RÉFÉRENCES BIBLIOGRAPHIQUES}

Bachelard G. (1948) - La Terre et les rêveries du repos, Paris, José Corti, $377 \mathrm{p}$.

Berrou P., Gouletquer P. (1973) - L'Épipaléolithique de la région de Plovan (Finistère), Bulletin de la Société préhistorique française, 70,6 , p. 166-172.

Biard M., Hinguant S. (2015) - Regards typotechnologique sur les productions lithiques foliaces du Solutréen de la vallée de l'Erve (Mayenne, France), Bulletin de la Société préhistorique française, 112, 2, p. 213-234.

Blanchet S., dir., avec la collaboration de Deloze V., Forre P., Pommier V. (2002) - Carnac (56) « La Croix Audran " Déviation sud des alignements - tranche 1, site $n^{\circ} 56034215$, document final de synthèse de sauvetage urgent, du 2 juillet au 14 septembre 2001, 65 p.

BoIsMoreau E. (1915) - Découverte de sculptures néolithiques et d'un abri-sous-roche aux Roches du Diable, près Quimperlé (Finistère). Séance du 25 mars 1915, Bulletin de la Société préhistorique française, 12, 3, p. 129-139.

Chancerel A., Paulet-Locard M.-A. (1991) - Le Mésolithique en Normandie, in Collectif, Mésolithique et néolithisation en France et dans les régions limitrophes, actes $\mathrm{du} 113^{\mathrm{e}}$ Congrès national des sociétés savantes (Strasbourg 1988), Paris, CTHS, p. 213-230.
Charraud F. (2013) - Espaces interculturels et évolution des systèmes techniques au Néolithique dans le Nord-Ouest de la France. Productions, usages et circulation des outillages en silex jurassiques de Normandie, thèse de troisième cycle, université de Nice, 2 vol., 480 et 538 p.

Chatellier P. DU (1888) - Le département du Finistère des temps préhistoriques à l'occupation romaine, Bulletin de la Société d'émulation des Côtes-du-Nord, 26, p. 52-114.

COLLECTIF (1984) - Les sites à microlithes entre Vilaine et marais Poitevin, Nantes, AEPHPL (supplément aux Études préhistoriques et protohistoriques des Pays de la Loire, 7), $300 \mathrm{p}$.

DÉCHelette J. (1908) - Manuel d'archéologie préhistorique, celtique et gallo-romaine, 1. Archéologie préhistorique, Paris, Picard, 747 p.

Duday H., Courtaud P., Robin K., Dujardin V., Gruet Y., Gouraud G., Martin H., San Juan-Foucher C. (1998) - La Vergne : La Grande Pièce (déviation de Saint-Jean d'Angély, Charente-Maritime), Bulletin de la Société préhistorique française, 95, 3, p. 423-435.

Foucher P., Wattez J., Gebhardt A., Musch J. (2000) - Les structures de combustion mésolithiques de la Pierre SaintLouis (Geay, Charente-Maritime), Paléo, 12, p. 165-200. 
GHeSQuiÈRE E. (2012) - Le Mésolithique en Basse-Normandie, thèse de doctorat, EHESS, Toulouse, 2 vol., 849 p.

Ghesquière E., Lefevre P., Marcigny C., Souffi B. (2000) - Le Mésolithique moyen du Nord Cotentin, BasseNormandie, France, Oxford, Archaeopress (BAR, International Series 856), $292 \mathrm{p}$.

Giot P.-R., Bordes F. (1955) - L'abri sous roche paléolithique de Grainfollet à Saint-Suliac (Ille-et-Vilaine), L'Anthropologie, 59, p. 205-234.

Giot P.-R., Hallegouet B., Monnier J.-L. (1977) - Le Paléolithique supérieur du pays de Léon (Finistère). Les gisements de Roc'h Toul, Parc ar Plenen (Guiclan), Enez Guennoc (Landéda) et la Forest-Landerneau, L'Anthropologie, 81, 2, p. 201-234.

Giot P.-R., Monnier J.-L. (1975) - Deux sites du Paléolithique moyen en Bretagne : le Mont-Dol et Bréhat-Goareva. Nouvelles données et comparaisons, Compte rendu de l'Académie des sciences (Paris), série $\mathrm{D}$, sciences naturelles, 280, p. 1433-1435.

Gouletquer P., Kayser O., Le Goffic M., Léopold P., Marchand G., Moullec J.-M. (1996) - Où sont passés les Mésolithiques côtiers bretons? Bilan 1985-1995 des prospections de surface dans le Finistère, Revue archéologique de l'Ouest, 13, p. 5-30.

Gouraud G. (1995) - Introduction au Préboréal du CentreOuest, in Épipaléolithique et Mésolithique du Sénonais et des régions voisines, actes du colloque (Passy, 20-21 novembre 1993), Sens, société archéologique de Sens (Cahiers, 2), p. 181-183.

GRUET M. (1984) - L'apport de deux sites angevins à la chronologie des terrasses fluviales: Roc-en-Pail en Chalonnes sur Loire et Port-Launay sur la Sarthe, Bulletin de l'association française pour l'étude du Quaternaire, 21, 1-3, p. 13-18.

Hallégouët B., Hinguant S., Gebhardt A., Monnier J.-L. (1992) - Le gisement paléolithique inférieur de Menez Drégan 1 (Plouhinec, Finistère). Premiers résultats des fouilles, Bulletin de la Société préhistorique française, 89, 3, p. 77-81.

Hinguant S., Moullé P.-E., Almudena A., Pigeaud R. (2012) - Pièces osseuses gravées du Solutréen moyen de la grotte Rochefort (Saint-Pierre-sur-Erve, Mayenne, France), Paléo, 23, p. 337-356.

JaCQuier J., NAudinot N. (2015) - Socio-Economic Significance of Stone Tools Recycling, Reuse and Maintenance at the End of the Lateglacial in Northwestern France, Quaternary International, 361, p. 269-287.

Juhel L., Ghesquière E., MarCigny C. (2006) - L'abri-sousroche Néolithique moyen de la Jupinerie (Omonville-laPetite, Manche), Internéo, 6, p. 55-66.

Juhel L., Marcigny C. (2008) - Les occupations du Néolithique moyen de l'abri sous roche de la Jupinerie (Omonville-laPetite, Manche), Revue de la Manche, 50, fasc. 200, p. 45-59.

Kelly R. L., Todd L . C. (1988) - Coming into the Country : Early Paleo-Indian Hunting and Mobility, American Antiquity, 53, 2, p. 231-244.

LAforge M., Monnier J.-L. (2011) - Contribution à la chronostratigraphie du gisement paléolithique inférieur de MenezDrégan 1 (Plouhinec, Finistère, France), Quaternaire, 22, 2, p. 91-104.
LAPlace-Jauretche G. (1957) - Les industries de Roc'h Toul et Par-ar-Plenen en Guiclan (Finistère), Bulletin de la Société préhistorique française, 54, 7-8, p. 422-438.

Le Goffic M. (1990)-L'abri-sous-roche de Pont-Glas en Plounéour-Ménez, Bulletin de la Société archéologique du Finistère, 119, p. 61-69.

LE Hir M. (1869) - Première grotte à silex taillés signalée en Bretagne, Matériaux pour l'histoire primitive et naturelle de l'homme, 5, p. 119-122.

Le Hir M. (1874) - Caverne de Roc'h Toul, en KerouguyIzella, commune de Guiclan (Finistère), Bulletin de la Société archéologique du Finistère, 1, p. 85-91.

Marchand G. (2005) - Les occupations mésolithiques à l'intérieur du Finistère. Bilan archéographique et méthodologique (2001-2003), Revue archéologique de l'Ouest, 22, p. $25-84$.

Marchand G. (2007) - Et maintenant, qu'est-ce qu'on fait? Le Mésolithique de l'Ouest, 80 ans après M. et S.-J. Péquart, in P. Buttin (dir.), Marthe et Saint-Just Péquart, archéologues des îles. De Houat à Hoëdic, 1923-1934, Île-d'Hoëdic, Melvan (La Revue des deux îles, 4), p. 213-228.

Marchand G. (2009) - The End of Mesolithic in Western France: From Taphonomy to the Understanding of Prehistoric Territories, in S. McCartan, R. Schulting, G. Warren et P. Woodman (dir.), Mesolithic Horizons, vol. 2, p. 507-513.

Marchand G. (2012) - Différences de potentiels géologiques entre massifs cristallins et bassins sédimentaires, in G. Marchand et G. Querré (dir), Roches et sociétés de la Préhistoire entre massifs cristallins et bassins sédimentaires, Rennes, Presses universitaires de Rennes (Archéologie et Cultures), p. 9-28.

Marchand G. (2014) - Préhistoire atlantique. Fonctionnement et évolution des sociétés du Paléolithique au Néolithique, Arles, Errance, 520 p.

Marchand G., Arthuis R., Philibert S., Sellami F., Sicard S., avec la collaboration de Forré P., LANOË S., NAUleaU J.-F., Quesnel L., Querré G. (2009) - Un habitat azilien en Anjou : les Chaloignes à Mozé-sur-Louet (Maine-etLoire), Gallia Préhistoire, 51, p. 1-113.

Marchand G., Blanchet S., Chevalier G., Gallais J.-Y., Le Goffic M., Naudinot N., Yven E. (2004) - La fin $\mathrm{du}$ Tardiglaciaire sur le Massif armoricain : territoires et cultures matérielles, Paléo, 16, p. 137-170.

Marchand G., Le Goffic M. (2009) - Give Us Some Small Sites Please! Report on the First Year of Excavations in the Rock Shelter of Pont-Glas (Plouneour-Menez, Finistère, France), Mesolithic Miscellany, 19, 2, p. 12-16.

Marchand G., Le Goffic M., Marcoux N. (2011) - Occupations mésolithiques fugaces dans l'abri-sous-roche de Pont-Glas : une analyse de la segmentation des chaînes opératoires dans l'espace pour évoquer la mobilité des groupes préhistoriques en Bretagne, in F. Bon, S. Costamagno et N. Valdeyron (dir.), Haltes de chasse en Préhistoire. Quelles réalités archéologiques?, actes du colloque international (Touluse, 13-15 mai 2009), P@lethnologie, 3, p. 313-333.

Marchand G., Monnier J.-L., Pustoc'h F., Querré L. (2014) - Un visage original du Tardiglaciaire en Bretagne : les occupations aziliennes dans l'abri-sous-roche de Kerbizien à Huelgoat, Paléo, 25, p. 5-45. 
Michel S. (2011) - Les premiers groupes mésolithiques de la France atlantique : enquête sur l'industrie lithique, thèse de doctorat, université Rennes 1, $536 \mathrm{p}$.

Monnier J.-L. (1980) - Le Paléolithique de la Bretagne dans son cadre géologique, Rennes, Travaux du laboratoire d'anthropologie, $607 \mathrm{p}$.

Monnier J.-L. (1988) - Organisation de l'espace des habitats paléolithiques en Bretagne, in Cultures et industries lithiques en milieu loessique, actes du colloque international (Amiens, 9-11 décembre 1986), Revue archéologique de Picardie, 1-2, p. 81-89.

MonNieR J.-L. (2006) - Les premiers peuplements de l'Ouest de la France. Cadre chronostratigraphique et paléoenvironnemental, Bulletin du musée d'anthropologie préhistorique de Monaco, 46, p. 3-20.

Monnier J.-L., Le Cloirec R. (1985) - Le gisement paléolithique inférieur de la Pointe de Saint-Colomban, Carnac (Morbihan), Gallia Préhistoire, 28, 1, p. 7-36.

Moullec J.-M. (1984) - Rapport de sondage archéologique, abri sous roche de Kerbizien (Huelgoat, 29), rapport d'opération, service régional de l'Archéologie de Bretagne, Rennes, $6 \mathrm{p}$

Moullec J.-M. (1985) - Rapport de sondage archéologique. Abri sous roche de Kerbizien. Site $n^{\circ}$ 29.02.16.081.002, service régional de l'Archéologie de Bretagne, Rennes, 8 p.

Naudinot N. (2010) - Dynamiques techno-économiques et de peuplement au Tardiglaciaire dans l'Ouest de la France, thèse de doctorat, université Rennes 1, $731 \mathrm{p}$.

Naudinot N. (2013a) - La fin du Tardiglaciaire dans le NordOuest de la France, Bulletin de la Société préhistorique française, 110, 2, p. 233-255.

Naudinot N. (2013b) - L'abri-sous-roche du Rocher de l'Impératrice à Plougastel-Daoulas, Finistère, rapport de sondage, service régional de l'Archéologie de Bretagne, Rennes, 98 p.

NAudinot N. (2014) - L'abri sous roche du Rocher de l'Impératrice à Plougastel-Daoulas, Finistère, rapport intermédiaire d'opération archéologique pluriannuelle, service régional de l'Archéologie de Bretagne, Rennes, 116 p.

NAUdinot N., JACQUier J. (2009) - Un site tardiglaciaire en place à la Fosse (Villiers-Charlemagne, Mayenne) : premiers résultats et implications chronoculturelles, Bulletin de la Société préhistorique française, 106, 1, p. 145-149.

NAudinot N., JacQuier J. (2013) - Le site Paléolithique final de la Fosse (Mayenne) : approche palethnologique d'une occupation de la transition Pléistocène-Holocène sur les bords de la Mayenne, rapport d'opération archéologique, service régional de l'Archéologie des Pays de la Loire, Nantes, $252 \mathrm{p}$

NAUdinot N., JACQUIER J. (2014) - Socio-economic Organization of Final Paleolithic societies: New Perspectives from an Aggregation Site in Western France, Journal of Anthropological Archaeology, 35, p. 177-189

Nicolas E., Marchand G., Deloze V., Juhel L., Vissac C., 2012 - Les occupations mésolithiques de Pen Hoat Salaün en Bretagne : premiers résultats de la fouille préventive et retour d'expérience sur les méthodes employées, Bulletin de la Société préhistorique française, 109, 3, p. 457-494.

Patre E. (1971) - Quelques sépultures du Poitou, du Mésolithique au Bronze moyen, Gallia Préhistoire, 14, 1, p. 139-244.
Plisson H. (2005) - Examen tracéologique des pointes aziliennes du Bois-Ragot, in A. Chollet et V. Dujardin (dir.), La Grotte du Bois-Ragot à Gouex (Vienne). Magdalénien et Azilien. Essais sur les hommes et leur environnement, Paris, Société préhistorique française (Mémoires, 38), p. 183-189.

Poulain A.-G. (1928) - Les stations ou gisements préhistoriques des environs de Vernon (Eure), Bulletin de la Société préhistorique de France, 25, 11, p. 464-474.

Rozoy J.-G. (1978) - Les derniers chasseurs. L'Épipaléolithique en France et en Belgique. Essai de synthèse, Reims, Société archéologique champenoise (Numéro spécial du Bulletin de la Société archéologique champenoise), 3 vol.

Soufri B. (2004) - Le Mésolithique en Haute-Normandie (France) L'exemple du site d'Acquigny «l'Onglais » (Eure) et sa contribution à l'étude des gisements mésolithiques de plein air, Oxford, John and Erica Hedges (BAR, International series 1307), 208 p.

Tinevez J.-Y.avec la collaboration de Gehan T. (1987) - Fouille de sauvetage d'un site à microlithes : les Pâtures à Villevêque (Maine-et-Loire), Études préhistoriques et historiques des Pays-de-la-Loire, p. 1-17.

Tolan-Smith C. (2003) - The Social Context of Landscape Larning, in M. Rockman et J. Steele (éd.) Colonization of Unfamiliar Landscapes: the Archaeology of Adaptation, Londres, Routledge, p. 116-29.

Van Vliet-lanoë B., Cliquet D., Auguste P., Folz E., Keen D., Schwenninger J.-L., Mercier N., Alix P. , Roupin Y., Meurisse M., Seignac H. (2006)-L'abri sous-roche du Rozel (France, Manche) : un habitat de la phase récente du Paléolithique moyen dans son contexte géomorphologique, Quaternaire, 17, 3, p. 207-258.

VISSET L. (1979) - Recherches palynologiques sur la végétation pléistocène et holocène de quelques sites du district phytogéographique de basse Loire, Nantes, Société des sciencnes naturelles de l'Ouest de la France (Supplément hors série au Bulletin de la société des sciences naturelles de l'Ouest de la France), 282 p.

Yven E. (2003a) - Kerhuellan (Plusquellec, Côtes-d'Armor), un « site-carrière " mésolithique, Revue archéologique de l'Ouest, 20, p. 33-51.

YvEN E. (2003b) - Exploitation des gîtes d'approvisionnement en matières premières lithiques au Mésolithique en Bretagne occidentale, in $\mathrm{F}$. Surmely (dir.), Les matières premières lithiques en Préhistoire, actes de la table ronde interantionale (Aurillac, 20-22 juin 2002), Cressensac, Association Préhistoire du SudOuest (Supplément à Préhistoire du Sud-Ouest, 5), p. 351-365.

Grégor MARChand

UMR 6566 « CREAAH », Archéosciences Bâtiment 24-25, université Rennes 1, CS74205, 35042 Rennes Cedex gregor.marchand@univ-rennes1.fr

Nicolas NaUdinot

UMR 7264 « CEPAM », université Nice Sophia Antipolis, campus Saint-Jean-d'Angély

24, av. des Diables Bleus, 06357 Nice Cedex nicolas.naudinot@cepam.cnrs.fr 